ESAIM: COCV 19 (2013) 91-111

DOI: $10.1051 / \mathrm{cocv} / 2011207$
ESAIM: Control, Optimisation and Calculus of Variations

www.esaim-cocv.org

\title{
MULTI-BUMP SOLUTIONS FOR NONLINEAR SCHRÖDINGER EQUATIONS WITH ELECTROMAGNETIC FIELDS
}

\author{
Huirong Pi ${ }^{1}$ and Chunhua WAng ${ }^{1}$
}

\begin{abstract}
In this paper, we are concerned with the existence of multi-bump solutions for a nonlinear Schrödinger equations with electromagnetic fields. We prove under some suitable conditions that for any positive integer $m$, there exists $\epsilon(m)>0$ such that, for $0<\epsilon<\epsilon(m)$, the problem has an $m$ bump complex-valued solution. As a result, when $\epsilon \rightarrow 0$, the equation has more and more multi-bump complex-valued solutions.
\end{abstract}

Mathematics Subject Classification. 35J10, 35B99, 35J60.

Received May 2, 2011. Revised November 30, 2011.

Published online 1st March 2012.

\section{INTRODUCTION}

In this paper, we are interested in the existence of multi-bump solutions for the following nonlinear problem

$$
\left(\frac{\nabla}{i}-A_{\epsilon}(x)\right)^{2} u+(1+\epsilon a(x)) u=|u|^{p-2} u, x \in \mathbb{R}^{N},
$$

where $2<p<\frac{2 N}{N-2}$ if $N \geq 3$ and $2<p<+\infty$ if $N=1$ or $N=2$ and $\epsilon>0$ is a parameter. $a(x)$ is a positive continuous function on $\mathbb{R}^{N}$, and $A_{\epsilon}=\left(A_{\epsilon, 1}(x), A_{\epsilon, 2}(x), \ldots, A_{\epsilon, N}(x)\right)$ is such that $A_{\epsilon, j}(x)(j=1,2, \ldots, N)$ is a real $C^{1}$ function on $\mathbb{R}^{N}$. Throughout this paper we assume that $a(x)$ and $A_{\epsilon}(x)$ satisfy the following conditions respectively:

$$
\begin{aligned}
& \left(\mathbf{H}_{\mathbf{1}}\right) \quad a(x) \in C\left(\mathbb{R}^{N}, \mathbb{R}^{+}\right), \lim _{|x| \rightarrow \infty} a(x)=0, \text { and } \lim _{|x| \rightarrow \infty} \frac{\ln (a(x))}{|x|}=0 ; \\
& \left(\mathbf{H}_{\mathbf{2}}\right) A_{\epsilon}(x)=\epsilon B(x) \text {, where } B(x) \in C^{1}\left(\mathbb{R}^{N}, \mathbb{R}^{N}\right) \text { is bounded. }
\end{aligned}
$$

Equation (1.1) rises in many fields of physics, in particular condensed matter physics and nonlinear optics (see [35])

$$
i \hbar \frac{\partial \Psi}{\partial t}=\left(\frac{\hbar}{i} \nabla-A(x)\right)^{2} \Psi+G(x) \Psi-f(x, \Psi),(t, x) \in \mathbb{R} \times \mathbb{R}^{N}(N \geq 2) .
$$

Keywords and phrases. Contraction map, electromagnetic fields, multi-bump solutions, nonlinear Schrödinger equation, variational reduction method.

1 School of Mathematics and Statistics, Central China Normal University, Wuhan 430079, P.R. China. wch5923@yahoo.com.cn 
The function $\Psi(x, t)$ takes on complex values, $\hbar$ is the Planck constant, $i$ is the imaginary unit. Here $A: \mathbb{R}^{N} \rightarrow$ $\mathbb{R}^{N}$ denotes a magnetic potential and the Schrödinger operator is defined by

$$
\left(\frac{\hbar}{i} \nabla-A(x)\right)^{2} \Psi:=-\hbar^{2} \triangle \Psi-\frac{2 \hbar}{i} A \cdot \nabla \Psi+|A|^{2} \Psi-\frac{\hbar}{i} \Psi \operatorname{div} A .
$$

Actually, in general dimension $N \geq 4$, the magnetic field $D$ is a 2 -form where $D_{k, j}=\partial_{j} A_{k}-\partial_{k} A_{j}$. In the case $N=3, D=\operatorname{curl} A$. The function $G: \mathbb{R}^{N} \rightarrow \mathbb{R}$ represents an electric potential.

We intend to find standing waves for $(1.2)$, namely solutions of the form $\Psi(x, t)=\mathrm{e}^{-\frac{\mathrm{i} E t}{\hbar}} u(x)$ for some function $u: \mathbb{R}^{N} \mapsto \mathbb{C}$. Substituting this ansatz into (1.2), one is led to solve the complex equation

$$
\left(\frac{\hbar}{i} \nabla-A(x)\right)^{2} u+V(x) u=f(x, u), x \in \mathbb{R}^{N},
$$

where $V(x)=G(x)-E$. If $\hbar=1, A(x)=A_{\epsilon}(x), V(x)=1+\epsilon a(x)$ and $f(x, u)=|u|^{p-2} u$, then (1.3) is reduced to (1.1). The transition from quantum mechanics to classical mechanics can be formally described by letting $\hbar \rightarrow 0$, and thus the existence of solutions for $\hbar$ small has physical interest. Standing waves for $\hbar$ small are usually referred as semi-classical bound states (see [22]).

When $A(x) \equiv 0,(1.3)$ reads

$$
-\hbar^{2} \triangle u+V(x) u=f(x, u), \quad x \in \mathbb{R}^{N} .
$$

In recent years, much attention has been paid to the study of the existence and uniqueness for one- or multibump bound states of (1.4). In [21], using a Lyapunov-Schmidt reduction, Floer and Weinstein established the existence of a standing wave solution of (1.4) when $N=1, f(x, u)=|u| u$ and $V(x)$ was a bounded function having a nondegenerate critical point for sufficiently small $\hbar$. Moreover, they showed that $u$ concentrated near the given non-degenerate critical point of $V$ when $\hbar$ tended to 0 . Their methods and results were later generalized by $\mathrm{Oh}[32,33]$ to the higher-dimensional case. For a potential $V$ without any nondegenerate critical point, Rabinowitz [34] obtained an existence result for (1.4) with $\hbar$ small, provided that $0<\inf _{x \in \mathbb{R}^{N}} V(x)<\liminf _{|x| \rightarrow \infty} V(x)$, using a global variational argument. These solutions concentrate near the global minima of $V$ as $\hbar \rightarrow 0$, as shown by Wang [38]. For more general case, one can see [40]. del Pino and Felmer [16,17] obtained multi-peak solutions having exactly $k$ maximum points provided that there were $k$ disjoint open bounded sets $\Lambda_{i}$ at its bottom. For more results concerning (1.4), see [7, 9, 10,18, 19,27,39].

When $A(x) \not \equiv 0$, we first mention a paper by Esteban and Lions [20], in which concentration-compactness principle of Lions was applied to solve some minimization problems under suitable assumptions on the magnetic field. Results concerning bounded vector potentials, when $V$ had a manifold of stationary points, were obtained by Cingolani and Secchi in [13] using a perturbation approach given by Ambrosetti et al. in [2]. Semiclassical multi-peak solutions for (1.3) for bounded vector potentials were constructed in [11] by Cao and Tang. In [14], using a penalization procedure (see [18]), Cingolani and Secchi extended the result in [13] to the case of a vector potential $A$, possibly unbounded. The penalization approach was also used by Bartsch et al. in [5], and later by Cingolani et al. in [15] to obtain multi-bump semiclassical bound for problem (1.2) with more general nonlinear term $f(x, \Psi)$. Concerning other papers on the topic, we mention that Kurata in [25] proved the existence of least energy solution of (1.3) for $\hbar>0$ under a condition relating $V(x)$ and $A(x)$. In [22,23], Helffer studied asymptotic behavior of the eigenfunctions of the Schrödinger operators with magnetic fields in the semiclassical limit. See also [6] for generalization of the results in [24] for potentials which were degenerate at infinity. For more related results, we can refer to $[3,12,15,36,37]$ and the references therein.

We should point out that in almost all papers listed above, the solutions obtained will concentrate around some points when the parameter which is the Planck constant $\hbar(\epsilon)$ tends to $0^{+}$. However, in this paper, we want to find solutions to (1.1) which do not concentrate near any point in the space. More precisely, we intend to look for solutions to (1.1) whose bumps are separated far apart and the distance between two bumps goes to infinity when $\epsilon \rightarrow 0$. Moreover, the size of each bump does not shrink and is fixed when $\epsilon \rightarrow 0$. This is greatly different 
from the concentration phenomenon described above. To this end, we use the idea introduced in the paper of Lin et al. [31], where $A_{\epsilon}(x) \equiv 0,(1.1)$ was considered as a real-valued problem and multi-bump real-valued solutions were found.

When $\epsilon \rightarrow 0$, the limiting equation of (1.1) is

$$
-\Delta u+u=|u|^{p-2} u, \quad u(x) \in H^{1}\left(\mathbb{R}^{N}, \mathbb{C}\right) .
$$

We will use the solutions of (1.5) to build up the approximate solutions for (1.1).

If we denote $U_{c}: \mathbb{R}^{N} \mapsto \mathbb{C}$ a least-energy solution to equation (1.5). By energy comparison (see [25]), one has that

$$
U_{c}(x)=\mathrm{e}^{\mathrm{i} \sigma} w\left(x-y_{0}\right),
$$

for some choice of $\sigma \in[0,2 \pi]$ and $y_{0} \in \mathbb{R}^{N}$, where $w \in C^{\infty}\left(\mathbb{R}^{N}, \mathbb{R}\right)$ is the unique solution of the problem

$$
\begin{cases}-\triangle u+u=u^{p-1}, & u>0 \text { in } \mathbb{R}^{N}, \\ u(x) \rightarrow 0, & \text { as }|x| \rightarrow+\infty .\end{cases}
$$

It is well-known that every positive solution of equation (1.6) has the form $w_{y}:=w(\cdot-y)$ for some $y \in \mathbb{R}^{N}$, $w$ satisfies, for some $c>0$,

$$
w(r) r^{\frac{N-1}{2}} \mathrm{e}^{r} \rightarrow 0, w^{\prime}(r) r^{\frac{N-1}{2}} \mathrm{e}^{r} \rightarrow-c, \text { as } r=|x| \rightarrow \infty,
$$

(see [26]). This solution $w$ will be employed as a building block to construct multi-bump solutions for (1.1). Let $m \geq 1$ be an integer. For sufficiently separated $y_{1}, y_{2}, \ldots, y_{m}$ in $\mathbb{R}^{N}$ and some choice of $\sigma \in[0,2 \pi]$, a solution of (1.1) which is close to $\sum_{j=1}^{m} \mathrm{e}^{\mathrm{i} \sigma} w\left(x-y_{j}\right):=\sum_{j=1}^{m} \eta w\left(x-y_{j}\right)$ in a sense which will be made clear later is called an $m$-bump solution.

For convenience, we denote

$$
V_{\epsilon}(x)=1+\epsilon a(x) .
$$

Let $E$ be a Hilbert space defined as the closure of $C_{0}^{\infty}\left(\mathbb{R}^{N}, \mathbb{C}\right)$ under the scalar product

$$
(u, v)_{\epsilon}=\operatorname{Re} \int\left(\frac{\nabla u}{i}-A_{\epsilon}(x) u\right) \overline{\left(\frac{\nabla v}{i}-A_{\epsilon}(x) v\right)}+V_{\epsilon}(x) u \bar{v} .
$$

The norm induced by the product $(\cdot, \cdot)_{\epsilon}$ is

$$
\begin{aligned}
\|u\|_{\epsilon} & =\left(\int\left|\frac{\nabla u}{i}-A_{\epsilon}(x) u\right|^{2}+V_{\epsilon}(x)|u|^{2}\right)^{\frac{1}{2}} \\
& =\left(\int|\nabla u|^{2}+\left|A_{\epsilon}(x)\right|^{2}|u|^{2}+V_{\epsilon}(x)|u|^{2}-2 \operatorname{Re} \int \frac{1}{i} \nabla u \cdot A_{\epsilon}(x) \bar{u}\right)^{\frac{1}{2}} .
\end{aligned}
$$

We use $\|\cdot\|$ and $(\cdot, \cdot)$ to denote the usual norm and inner product of $H^{1}\left(\mathbb{R}^{N}, \mathbb{C}\right)$. By the assumptions of $A_{\epsilon}(x)$ and $a(x)$ and Lemma A.2 we know that $\|\cdot\|_{\epsilon}$ in $E$ is equivalent to $\|\cdot\|$ in $H^{1}\left(\mathbb{R}^{N}, \mathbb{C}\right)$. The energy functional associated with (1.1) is defined by

$$
I_{\epsilon}(u)=\frac{1}{2} \int\left|\frac{\nabla u}{i}-A_{\epsilon}(x) u\right|^{2}+V_{\epsilon}(x)|u|^{2}-\frac{1}{p} \int|u|^{p}, \forall u \in E .
$$

Denote the functional related to $(1.5)$ by $I_{0}(u)$, that is

$$
I_{0}(u)=\frac{1}{2} \int\left(|\nabla u|^{2}+|u|^{2}\right)-\frac{1}{p} \int|u|^{p}, \forall u \in H^{1}\left(\mathbb{R}^{N}, \mathbb{C}\right) .
$$


Let

$$
Z=\left\{\mathrm{e}^{\mathrm{i} \sigma} w(x) ;(x, \sigma) \in \mathbb{R}^{N} \times[0,2 \pi] \simeq \mathbb{R}^{N} \times S^{1}\right\} .
$$

From $[13,14]$, we know that $Z$ is non-degenerate, that is

$$
\operatorname{ker}_{0}^{\prime \prime}(\eta w)=\operatorname{span}_{\mathbb{R}}\left\{\frac{\partial(\eta w)}{\partial x_{1}}, \ldots, \frac{\partial(\eta w)}{\partial x_{N}}, \frac{\partial(\eta w)}{\partial \sigma}=i \eta w\right\} .
$$

Our main result is as follows:

Theorem 1.1. Let $\left(H_{1}\right)$ and $\left(H_{2}\right)$ hold. Then for any positive integer $m$ there exists $\epsilon(m)>0$ such that for $0<\epsilon<\epsilon(m)$, problem (1.1) has an $m$-bump complex-valued solution $u$ with the following form:

$$
u=\sum_{j=1}^{m} \mathrm{e}^{\mathrm{i} \sigma} w\left(x-y_{j}^{\epsilon}\right)+v_{\epsilon, \sigma, y^{\epsilon}},
$$

where $\sigma$ is any constant in $[0,2 \pi], y^{\epsilon}=\left(y_{1}^{\epsilon}, y_{2}^{\epsilon}, \ldots, y_{m}^{\epsilon}\right) \in\left(\mathbb{R}^{N}\right)^{m}$ with $\left|y_{i}^{\epsilon}-y_{j}^{\epsilon}\right| \rightarrow+\infty$ as $\epsilon \rightarrow 0$ for any $i \neq j$, $\left\|v_{\epsilon, \sigma, y^{\epsilon}}\right\|_{\epsilon}=O(\epsilon)$.

Remark: By the very similar argument, we can obtain the following result (see also [30]):

Suppose that $A_{\epsilon}(x)$ satisfies $\left(H_{2}\right)$ and $a(x)$ satisfies

$$
\left(\mathbf{H}_{1}^{\prime}\right) a(x) \in C\left(\mathbb{R}^{N}, \mathbb{R}^{+}\right), \lim _{|x| \rightarrow \infty} a(x)=0, a(x) \geq c \mathrm{e}^{-\delta|x|} \text { for some } c>0, \delta>0 .
$$

If $m \in \mathbb{N}$ satisfies $m<1+\frac{p-2}{2 \delta(p-1)}$, then there exists $\epsilon(m)>0$ such that for $0<\epsilon<\epsilon(m)$, the following equation

$$
\left(\frac{\nabla}{i}-A_{\epsilon}(x)\right)^{2} u+u=(1-\epsilon a(x))|u|^{p-2} u, x \in \mathbb{R}^{N},
$$

has an $m$-bump complex-valued solution.

Moreover, if $A_{\epsilon}(x)$ satisfies $\left(H_{2}\right)$ and $a(x) \in C\left(\mathbb{R}^{N}, \mathbb{R}^{+}\right)$satisfies

$$
\left(\mathbf{H}_{1}^{\prime \prime}\right) \lim _{|x| \rightarrow \infty} a(x)=0 \text {, and there exists } c>0 \text { such that } a(x) \geq c \mathrm{e}^{-\delta|x|}, \forall \delta>0 .
$$

Then for any $m \in \mathbb{N}$, there exists $\epsilon(m)>0$ such that for $0<\epsilon<\epsilon(m)$, (1.8) has an $m$-bump complex-valued solution. As a consequence, when $\epsilon \rightarrow 0,(1.8)$ has more and more multi-bump complex-valued solutions.

We mainly use the variational reduction method to prove Theorem 1.1. Our argument is partially inspired by [28-31]. We first reduce the problem to look for solutions of (1.1) to the problem to find the critical points of a function defined on a open subset of a finite dimensional Euclidian space. Then we prove that the function achieves its maximum at the interior of that open subset. We remark that differently from [28,30,31], we need to overcome many additional difficulties which arise because of the appearance of the magnetic field $A_{\epsilon}(x)$. Problem (1.1) cannot be changed into a pure real-valued problem, hence we should deal with a complexvalued problem directly, which causes more new difficulties in employing the methods in dealing with singularly perturbed problems (see [1]).

This paper is organized as follows. In Section 2, we will carry out the reduction. Then, we will study the reduced finite dimensional problem and prove Theorem 1.1 in Section 3. In Appendix A, we give some elementary inequalities which are useful in our estimates.

\section{Notation:}

1. We simply write $\int f$ to mean the Lebesgue integral of $f(x)$ in $\mathbb{R}^{N}$;

2. the complex conjugate of any number $z \in \mathbb{C}$ will be denoted by $\bar{z}$;

3. the real part of a number $z \in \mathbb{C}$ will be denoted by $\operatorname{Re} z$;

4. the ordinary inner product between two vectors $a, b \in \mathbb{R}^{N}$ will be denoted by $a \cdot b$;

5. $C, c_{i}, C_{i}, C_{i}^{\prime}(i=1,2, \ldots)$ denote generic constants, which may vary inside a chain of inequalities. 


\section{VARIATIONAL REDUCTION}

Fix $m \in \mathbb{N}$. For $\lambda>0$ and $m \geq 2$, define

$$
\Omega_{\lambda}=\left\{\left(y_{1}, y_{2}, \ldots, y_{m}\right) \in\left(\mathbb{R}^{N}\right)^{m}:\left|y_{k}-y_{j}\right|>\lambda, \text { for any } k \neq j\right\} .
$$

For simplicity, we make the convention

$$
\Omega_{\lambda} \equiv \mathbb{R}^{N}(\forall \lambda>0)
$$

if $m=1$. For $y \in \Omega_{\lambda}$, let

$$
z_{y}=\sum_{j=1}^{m} \mathrm{e}^{\mathrm{i} \sigma} w\left(x-y_{j}\right)=\sum_{j=1}^{m} \eta w_{y_{j}}=\eta u_{y},
$$

where $w_{y_{j}}=w\left(\cdot-y_{j}\right), u_{y}=\sum_{j=1}^{m} w_{y_{j}}$.

Let $y \in \Omega_{y}$. Define

$$
W_{y}=\left\{v \in E \mid \operatorname{Re} \int \eta w_{y_{j}}^{p-2} \frac{\partial w_{y_{j}}}{\partial x_{\alpha}} \bar{v}=0 \text { and } \operatorname{Re} \int \eta w_{y_{j}}^{p-1} \bar{v}=0\right\}
$$

where $\alpha=1,2, \ldots, N$ and $j=1,2, \ldots, m$.

It is easy to check that

$$
\begin{aligned}
& \operatorname{Re} \int\left[\left(\frac{\nabla}{i}-A_{\epsilon}(x)\right) v_{1}\right] \overline{\left(\frac{\nabla}{i}-A_{\epsilon}(x)\right) v_{2}}+\operatorname{Re} \int V_{\epsilon}(x) v_{1} \bar{v}_{2} \\
& -\left[(p-2) \operatorname{Re} \int\left|z_{y}\right|^{p-4} \operatorname{Re}\left(z_{y} \bar{v}_{2}\right) z_{y} \bar{v}_{1}+\int\left|z_{y}\right|^{p-2} \operatorname{Re}\left(v_{1} \bar{v}_{2}\right)\right], \forall v_{1}, v_{2} \in W_{y}
\end{aligned}
$$

is a bounded bi-linear functional in $W_{y}$. Hence there is a bounded linear operator $L_{y}$ from $W_{y}$ to $W_{y}$, such that

$$
\begin{aligned}
\left\langle L_{y} v_{1}, v_{2}\right\rangle= & \operatorname{Re} \int\left[\left(\frac{\nabla}{i}-A_{\epsilon}(x)\right) v_{1}\right] \overline{\left(\frac{\nabla}{i}-A_{\epsilon}(x)\right) v_{2}}+\operatorname{Re} \int V_{\epsilon}(x) v_{1} \bar{v}_{2} \\
& -\left[(p-2) \operatorname{Re} \int\left|z_{y}\right|^{p-4} \operatorname{Re}\left(z_{y} \bar{v}_{2}\right) z_{y} \bar{v}_{1}+\int\left|z_{y}\right|^{p-2} \operatorname{Re}\left(v_{1} \bar{v}_{2}\right)\right], \forall v_{1}, v_{2} \in W_{y} .
\end{aligned}
$$

The following lemma shows that $L_{y}$ is invertible in $W_{y}$.

Lemma 2.1. There exist positive constants $\lambda_{0}, \epsilon_{0}$ and $\zeta_{0}$ such that for any $\lambda>\lambda_{0}, 0<\epsilon<\epsilon_{0}, \sigma \in[0,2 \pi], y \in \Omega_{\lambda}$ and $v \in W_{y}$,

$$
\left\|L_{y} v\right\|_{\epsilon} \geq \zeta_{0}\|v\|_{\epsilon} .
$$

Proof. We argue by contradiction argument. Suppose that there exist $\left\{y_{k, n}\right\}_{n=1}^{\infty} \subset \mathbb{R}^{N}, k=1,2, \ldots, m$, with $\left|y_{k, n}-y_{j, n}\right| \rightarrow \infty$ for $k \neq j$ and $v_{n} \in W_{y_{n}}$ with $\left\|v_{n}\right\|_{\epsilon}=1$ such that

$$
\left\|L_{y_{n}} v_{n}\right\|_{\epsilon}=o(1)\left\|v_{n}\right\|_{\epsilon}=o(1)
$$

where $y_{n}=\left(y_{1, n}, y_{2, n}, \ldots, y_{m, n}\right)$. Without loss of generality, we may assume that $\left|y_{k, n}\right| \rightarrow \infty, k=1,2, \ldots, m$ as $n \rightarrow \infty$. Assume that

$$
v_{n}\left(\cdot+y_{k, n}\right) \rightarrow v_{k}^{*}, \text { in } E, k=1,2, \ldots, m, \text { as } n \rightarrow \infty
$$

and

$$
v_{n}\left(\cdot+y_{k, n}\right) \rightarrow v_{k}^{*}, \text { strongly in } L_{l o c}^{2}\left(\mathbb{R}^{N}\right), \quad k=1,2, \ldots, m, \text { as } n \rightarrow \infty .
$$


From

$$
\operatorname{Re} \int \eta w_{y_{k, n}}^{p-2} \frac{\partial w_{y_{k, n}}}{\partial x_{\alpha}} \bar{v}_{n}=0 \text { and } \operatorname{Re} \int \eta w_{y_{k, n}}^{p-1} \bar{v}_{n}=0
$$

for $\alpha=1,2, \ldots, N$ and $k=1,2, \ldots, m$, we obtain

$$
\operatorname{Re} \int \eta w^{p-2} \frac{\partial w}{\partial x_{\alpha}} \overline{v_{n}\left(\cdot+y_{k, n}\right)}=0 \text { and } \operatorname{Re} \int \eta w^{p-1} \overline{v_{n}\left(\cdot+y_{k, n}\right)}=0
$$

for $\alpha=1,2, \ldots, N$ and $k=1,2, \ldots, m$. So $v_{k}^{*}$ satisfies

$$
\operatorname{Re} \int \eta w^{p-2} \frac{\partial w}{\partial x_{\alpha}} \overline{v_{k}^{*}}=0 \text { and } \operatorname{Re} \int \eta w^{p-1} \overline{v_{k}^{*}}=0
$$

for $\alpha=1,2, \ldots, N$ and $k=1,2, \ldots, m$.

Now we prove that $v_{k}^{*} \in \operatorname{ker} I_{0}^{\prime \prime}(\eta w)$, that is

$$
\operatorname{Re} \int \nabla v_{k}^{*} \nabla \bar{\varphi}+\operatorname{Re} \int v_{k}^{*} \bar{\varphi}-\left[(p-2) \operatorname{Re} \int \eta w^{p-3} \operatorname{Re}(\eta w \bar{\varphi}) \overline{v_{k}^{*}}+\operatorname{Re} \int w^{p-2} \operatorname{Re}\left(v_{k}^{*} \bar{\varphi}\right)\right]=0, \quad \forall \varphi \in E .
$$

Define

$$
\widetilde{W}_{y}=\left\{\varphi: \varphi \in E, \operatorname{Re} \int \eta w^{p-2} \frac{\partial w}{\partial x_{\alpha}} \bar{\varphi}=0 \text { and } \operatorname{Re} \int \eta w^{p-1} \bar{\varphi}=0, \alpha=1,2, \ldots, N\right\} .
$$

Note that

$$
\begin{aligned}
o(1)\|\varphi\|= & \left\langle L_{y_{n}} v_{n}, \varphi\right\rangle \\
= & \operatorname{Re} \int\left[\left(\frac{\nabla}{i}-A_{\epsilon}(x)\right) v_{n}\right] \overline{\left(\frac{\nabla}{i}-A_{\epsilon}(x)\right) \varphi}+\operatorname{Re} \int V_{\epsilon}(x) v_{n} \bar{\varphi} \\
& -\left[(p-2) \operatorname{Re} \int\left|z_{y_{n}}\right|^{p-4} \operatorname{Re}\left(z_{y_{n}} \bar{\varphi}\right) z_{y_{n}} \bar{v}_{n}+\int\left|z_{y_{n}}\right|^{p-2} \operatorname{Re}\left(v_{n} \bar{\varphi}\right)\right], \quad \forall \varphi \in \widetilde{W}_{y} .
\end{aligned}
$$

Let $\varphi \in C_{0}^{\infty}\left(\mathbb{R}^{N}, \mathbb{C}\right) \cap \widetilde{W}_{y}$ and take $\varphi_{n}(x)=: \varphi\left(x+y_{k, n}\right) \in C_{0}^{\infty}\left(\mathbb{R}^{N}, \mathbb{C}\right)$. Inserting $\varphi_{n}$ into (2.4) and choosing $\epsilon>0$ small enough and $\lambda>0$ big enough, we find

$$
\operatorname{Re} \int \nabla v_{k}^{*} \nabla \bar{\varphi}+\operatorname{Re} \int v_{k}^{*} \bar{\varphi}-\left[(p-2) \operatorname{Re} \int \eta w^{p-3} \operatorname{Re}(\eta w \bar{\varphi}) \overline{v_{k}^{*}}+\operatorname{Re} \int w^{p-2} \operatorname{Re}\left(v_{k}^{*} \bar{\varphi}\right)\right]=0
$$

Since $C_{0}^{\infty}\left(\mathbb{R}^{N}, \mathbb{C}\right)$ is dense in $H^{1}\left(\mathbb{R}^{N}, \mathbb{C}\right)$ and the norm $\|\cdot\|_{\epsilon}$ in $E$ is equivalent to $\|\cdot\|$ in $H^{1}\left(\mathbb{R}^{N}, \mathbb{C}\right), C_{0}^{\infty}\left(\mathbb{R}^{N}, \mathbb{C}\right)$ is dense in $E$. It is easy to show that

$$
\operatorname{Re} \int \nabla v_{k}^{*} \nabla \bar{\varphi}+\operatorname{Re} \int v_{k}^{*} \bar{\varphi}-\left[(p-2) \operatorname{Re} \int \eta w^{p-3} \operatorname{Re}(\eta w \bar{\varphi}) \overline{v_{k}^{*}}+\operatorname{Re} \int w^{p-2} \operatorname{Re}\left(v_{k}^{*} \bar{\varphi}\right)\right]=0, \quad \forall \varphi \in \widetilde{W}_{y}
$$


But (2.5) holds for $\varphi=c_{0} \eta w+\sum_{\alpha=1}^{N} c_{\alpha} \frac{\partial(\eta w)}{\partial x_{\alpha}}$. Hence (2.5) is true for any $\varphi \in E$, which means that $v_{k}^{*} \in$ $\operatorname{ker} I_{0}^{\prime \prime}(\eta w)$ and hence $v_{k}^{*}=c_{0} \eta w+\sum_{\alpha=1}^{N} c_{\alpha} \frac{\partial(\eta w)}{\partial x_{\alpha}}$. From (2.3), we find

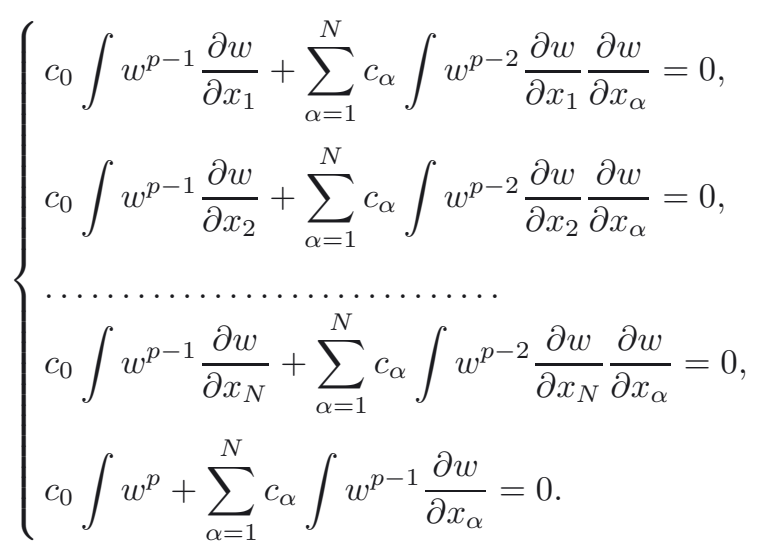

Consequently, $c_{0}=c_{1}=\ldots=c_{N}=0$ and $v_{k}^{*}=0$. As a result,

$$
\int_{B_{R}(0)}\left|v_{n}\right|^{2}=o(1) \text {, for any } R>0 .
$$

Thus, choosing $\epsilon>0$ small enough, we have

$$
\begin{aligned}
\left\langle L_{y_{n}} v_{n}, v_{n}\right\rangle & =\int\left\{\left|\left(\frac{\nabla}{i}-A_{\epsilon}(x)\right) v_{n}\right|^{2}+V_{\epsilon}(x)\left|v_{n}\right|^{2}-\left[(p-2)\left|z_{y_{n}}\right|^{p-4}\left(\operatorname{Re}\left(z_{y_{n}} \bar{v}_{n}\right)\right)^{2}+\left|z_{y_{n}}\right|^{p-2}\left|v_{n}\right|^{2}\right]\right\} \\
& \geq \int\left[\left|\left(\frac{\nabla}{i}-A_{\epsilon}(x)\right) v_{n}\right|^{2}+V_{\epsilon}(x)\left|v_{n}\right|^{2}\right]-(p-1) \int\left|z_{y_{n}}\right|^{p-2}\left|v_{n}\right|^{2} \\
& =\left\|v_{n}\right\|_{\epsilon}^{2}-(p-1) \int\left|z_{y_{n}}\right|^{p-2}\left|v_{n}\right|^{2} \\
& =1+O\left(\mathrm{e}^{-(p-2) R}\right) \int_{B_{R}(0)}\left|v_{n}\right|^{2} \geq \frac{1}{2}+o(1) .
\end{aligned}
$$

This is a contradiction to $(2.2)$.

Let

$$
J(\varphi)=I_{\epsilon}\left(z_{y}+\varphi\right), \varphi \in W_{y} .
$$

We have the following result:

Lemma 2.2. There exist positive constants $\epsilon_{0}$ and $\lambda_{0}$ such that for $0<\epsilon<\epsilon_{0}$ and $\lambda \geq \lambda_{0}$, there exists a $C^{1}$ map

depending on $\lambda$ and $\epsilon$, satisfying

$$
v_{\lambda, \epsilon}: \Omega_{\lambda} \times[0,2 \pi] \rightarrow E
$$

(i) for any $(y, \sigma) \in \Omega_{\lambda} \times[0,2 \pi], v_{\lambda, \epsilon, \sigma, y} \in W_{y}$ and

$$
\left\langle\frac{\partial J\left(v_{\lambda, \epsilon, \sigma, y}\right)}{\partial v_{\lambda, \epsilon, \sigma, y}}, \varphi\right\rangle=0, \forall \varphi \in W_{y}
$$

(ii)

$$
\left\|v_{\lambda, \epsilon, \sigma, y}\right\|_{\epsilon} \leq \epsilon^{1-\tau}+\sum_{k \neq j} \mathrm{e}^{-\frac{p-1-\tau}{p}\left|y_{k}-y_{j}\right|}
$$

where $\tau>0$ is an arbitrary small constant. Moreover, $v_{\lambda, \epsilon, \sigma, y}=\mathrm{e}^{\mathrm{i} \sigma} V_{\lambda, \epsilon, y}$ with $V_{\lambda, \epsilon, y} \in E$ independent of $\sigma$. 
Proof. Noting that

$$
\operatorname{Re} \int \nabla z_{y} \nabla \bar{v}_{\lambda, \epsilon, \sigma, y}+\operatorname{Re} \int z_{y} \bar{v}_{\lambda, \epsilon, \sigma, y}=\sum_{j=1}^{m} \operatorname{Re} \int \eta w_{y_{j}}^{p-1} \bar{v}_{\lambda, \epsilon, \sigma, y}
$$

we see

$$
\begin{aligned}
J\left(v_{\lambda, \epsilon, \sigma, y}\right)= & I_{\epsilon}\left(z_{y}+v_{\lambda, \epsilon, \sigma, y}\right) \\
= & \frac{1}{2} \int\left[\left|\left(\frac{\nabla}{i}-A_{\epsilon}(x)\right)\left(z_{y}+v_{\lambda, \epsilon, \sigma, y}\right)\right|^{2}+V_{\epsilon}(x)\left|z_{y}+v_{\lambda, \epsilon, \sigma, y}\right|^{2}\right]-\frac{1}{p} \int\left|z_{y}+v_{\lambda, \epsilon, \sigma, y}\right|^{p} \\
= & \left\{\frac{1}{2} \int\left|\left(\frac{\nabla}{i}-A_{\epsilon}(x)\right) z_{y}\right|^{2}+V_{\epsilon}(x)\left|z_{y}\right|^{2}-\frac{1}{p} \int\left|z_{y}\right|^{p}\right\}+\left\{\operatorname{Re} \int V_{\epsilon}(x) z_{y} \bar{v}_{\lambda, \epsilon, \sigma, y}\right. \\
& \left.+\operatorname{Re} \int\left(\frac{\nabla}{i}-A_{\epsilon}(x)\right) z_{y} \overline{\left(\frac{\nabla}{i}-A_{\epsilon}(x)\right) v_{\lambda, \epsilon, \sigma, y}}-\operatorname{Re} \int\left|z_{y}\right|^{p-2} z_{y} \bar{v}_{\lambda, \epsilon, \sigma, y}\right\} \\
& +\left\{\frac{1}{2} \int\left|\left(\frac{\nabla}{i}-A_{\epsilon}(x)\right) v_{\lambda, \epsilon, \sigma, y}\right|^{2}+V_{\epsilon}(x)\left|v_{\lambda, \epsilon, \sigma, y}\right|^{2}\right. \\
& \left.-\left[(p-2)\left|z_{y}\right|^{p-4}\left(\operatorname{Re}\left(z_{y} \bar{v}_{\lambda, \epsilon, \sigma, y}\right)\right)^{2}+\left|z_{y}\right|^{p-2}\left|v_{\lambda, \epsilon, \sigma, y}\right|^{2}\right]\right\} \\
& -\left\{\frac{1}{p} \int\left[\left|z_{y}+v_{\lambda, \epsilon, \sigma, y}\right|^{p}-\left|z_{y}\right|^{p}-p \operatorname{Re}\left(\left|z_{y}\right|^{p-2} z_{y} \bar{v}_{\lambda, \epsilon, \sigma, y}\right)\right]\right. \\
& \left.-\frac{p}{2}\left[(p-2)\left|z_{y}\right|^{p-4}\left(\operatorname{Re}\left(z_{y} \bar{v}_{\lambda, \epsilon, \sigma, y}\right)\right)^{2}+\left|z_{y}\right|^{p-2}\left|v_{\lambda, \epsilon, \sigma, y}\right|^{2}\right]\right\} .
\end{aligned}
$$

Direct calculation yields

$$
\begin{aligned}
& \operatorname{Re} \int\left(\frac{\nabla}{i}-A_{\epsilon}(x)\right) z_{y} \overline{\left(\frac{\nabla}{i}-A_{\epsilon}(x)\right) v_{\lambda, \epsilon, \sigma, y}}= \operatorname{Re} \int \nabla z_{y} \nabla \bar{v}_{\lambda, \epsilon, \sigma, y}-\epsilon \operatorname{Re} \int \frac{\nabla}{i} z_{y} B(x) \bar{v}_{\lambda, \epsilon, \sigma, y} \\
&-\epsilon \operatorname{Re} \int B(x) z_{y} \frac{\bar{\nabla}}{i} v_{\lambda, \epsilon, \sigma, y} \\
&+\epsilon^{2} \operatorname{Re} \int|B(x)|^{2} z_{y} \bar{v}_{\lambda, \epsilon, \sigma, y}
\end{aligned}
$$

Hence,

$$
J\left(v_{\lambda, \epsilon, \sigma, y}\right)=: J(0)+l_{y}\left(v_{\lambda, \epsilon, \sigma, y}\right)+\frac{1}{2}\left\langle L_{y} v_{\lambda, \epsilon, \sigma, y}, v_{\lambda, \epsilon, \sigma, y}\right\rangle-R_{y}\left(v_{\lambda, \epsilon, \sigma, y}\right),
$$

where

$$
\begin{aligned}
l_{y}\left(v_{\lambda, \epsilon, \sigma, y}\right)= & \sum_{j=1}^{m} \operatorname{Re} \int \eta w_{y_{j}}^{p-1} \bar{v}_{\lambda, \epsilon, \sigma, y}-\operatorname{Re} \int\left|z_{y}\right|^{p-2} z_{y} \bar{v}_{\lambda, \epsilon, \sigma, y} \\
& -\epsilon \operatorname{Re} \int \frac{\nabla}{i} z_{y} B(x) \bar{v}_{\lambda, \epsilon, \sigma, y}-\epsilon \operatorname{Re} \int B(x) z_{y} \frac{\bar{\nabla}}{i} v_{\lambda, \epsilon, \sigma, y} \\
& +\epsilon^{2} \operatorname{Re} \int|B(x)|^{2} z_{y} \bar{v}_{\lambda, \epsilon, \sigma, y}+\epsilon \operatorname{Re} \int a(x) z_{y} \bar{v}_{\lambda, \epsilon, \sigma, y} .
\end{aligned}
$$


$L_{y}$ is the bounded linear map from $W_{y}$ to $W_{y}$ in Lemma 2.1, and

$$
\begin{aligned}
R_{y}\left(v_{\lambda, \epsilon, \sigma, y}\right)= & \frac{1}{p} \int\left\{\left[\left|z_{y}+v_{\lambda, \epsilon, \sigma, y}\right|^{p}-\left|z_{y}\right|^{p}-p \operatorname{Re}\left(\left|z_{y}\right|^{p-2} z_{y} \bar{v}_{\lambda, \epsilon, \sigma, y}\right)\right]\right. \\
& \left.-\frac{p}{2}\left[(p-2)\left|z_{y}\right|^{p-4}\left(\operatorname{Re}\left(z_{y} \bar{v}_{\lambda, \epsilon, \sigma, y}\right)\right)^{2}+\left|z_{y}\right|^{p-2}\left|v_{\lambda, \epsilon, \sigma, y}\right|^{2}\right]\right\} .
\end{aligned}
$$

It is easy to check that $l_{y}\left(v_{\lambda, \epsilon, \sigma, y}\right)$ is a bounded linear functional in $W_{y}$, so there exists an $l_{y, k} \in W_{y}$ such that

$$
l_{y}\left(v_{\lambda, \epsilon, \sigma, y}\right)=\left\langle l_{y, k}, v_{\lambda, \epsilon, \sigma, y}\right\rangle .
$$

Thus, to find a critical point for $J\left(v_{\lambda, \epsilon, \sigma, y}\right)$, we only need to solve

$$
l_{y, k}+L_{y} v_{\lambda, \epsilon, \sigma, y}-R_{y}^{\prime}\left(v_{\lambda, \epsilon, \sigma, y}\right)=0 .
$$

Lemma 2.1 implies that $L_{y}$ is invertible. Thus, (2.7) can be rewritten as

$$
v_{\lambda, \epsilon, \sigma, y}=A_{y}\left(v_{\lambda, \epsilon, \sigma, y}\right)=:-L_{y}^{-1} l_{y, k}+L_{y}^{-1} R_{y}^{\prime}\left(v_{\lambda, \epsilon, \sigma, y}\right) .
$$

Set

$$
S_{y}=\left\{v_{\lambda, \epsilon, \sigma, y}: v_{\lambda, \epsilon, \sigma, y} \in W_{y},\left\|v_{\lambda, \epsilon, \sigma, y}\right\|_{\epsilon} \leq \epsilon^{1-\tau}+\sum_{k \neq j} \mathrm{e}^{-\frac{p-1-\tau}{p}\left|y_{k}-y_{j}\right|}\right\} .
$$

If $2<p \leq 3$, we can check that

$$
\left\|R_{y}^{\prime}\left(v_{\lambda, \epsilon, \sigma, y}\right)\right\|_{\epsilon} \leq C\left\|v_{\lambda, \epsilon, \sigma, y}\right\|_{\epsilon}^{p-1} \text { and }\left\|R_{y}^{\prime \prime}\left(v_{\lambda, \epsilon, \sigma, y}\right)\right\|_{\epsilon} \leq C\left\|v_{\lambda, \epsilon, \sigma, y}\right\|_{\epsilon}^{p-2} .
$$

Thus,

$$
\begin{aligned}
\left\|A_{y}\left(v_{\lambda, \epsilon, \sigma, y}^{1}\right)-A\left(v_{\lambda, \epsilon, \sigma, y}^{2}\right)\right\|_{\epsilon} & =\left\|L_{y}^{-1} R_{y}^{\prime}\left(v_{\lambda, \epsilon, \sigma, y}^{1}\right)-L_{y}^{-1} R_{y}^{\prime}\left(v_{\lambda, \epsilon, \sigma, y}^{2}\right)\right\|_{\epsilon} \\
& \leq C\left\|R_{y}^{\prime}\left(v_{\lambda, \epsilon, \sigma, y}^{1}\right)-R_{y}^{\prime}\left(v_{\lambda, \epsilon, \sigma, y}^{2}\right)\right\|_{\epsilon} \\
& \leq C\left\|R_{y}^{\prime \prime}\left(\theta v_{\lambda, \epsilon, \sigma, y}^{1}+(1-\theta) v_{\lambda, \epsilon, \sigma, y}^{2}\right)\right\|_{\epsilon}\left\|v_{\lambda, \epsilon, \sigma, y}^{1}-v_{\lambda, \epsilon, \sigma, y}^{2}\right\|_{\epsilon} \\
& \leq C\left(\left\|v_{\lambda, \epsilon, \sigma, y}^{1}\right\|_{\epsilon}^{p-2}+\left\|v_{\lambda, \epsilon, \sigma, y}^{2}\right\|_{\epsilon}^{p-2}\right)\left\|v_{\lambda, \epsilon, \sigma, y}^{1}-v_{\lambda, \epsilon, \sigma, y}^{2}\right\|_{\epsilon} \\
& \leq \frac{1}{2}\left\|v_{\lambda, \epsilon \sigma, y}^{1}-v_{\lambda, \epsilon, \sigma, y}^{2}\right\|_{\epsilon},
\end{aligned}
$$

where $\theta \in(0,1)$.

Thus, we have proved that if $2<p \leq 3, A_{y}$ is a contraction map.

When $\epsilon \rightarrow 0$ and $\lambda \rightarrow \infty$, Lemma 2.3 below implies that

$$
\left\|l_{y, k}\right\|_{\epsilon} \leq C\left(\epsilon+\sum_{k \neq j} \mathrm{e}^{-\frac{p-1}{p}\left|y_{k}-y_{j}\right|}\right) .
$$

Hence,

$$
\begin{aligned}
\left\|A_{y}\left(v_{\lambda, \epsilon, \sigma, y}\right)\right\|_{\epsilon} & =\left\|A_{y}\left(v_{\lambda, \epsilon, \sigma, y}\right)-A_{y}(0)\right\|_{\epsilon}+\left\|A_{y}(0)\right\|_{\epsilon} \\
& \leq C \epsilon^{p-2}\left\|v_{\lambda, \epsilon, \sigma, y}\right\|_{\epsilon}+C\left\|l_{y, k}\right\|_{\epsilon} \\
& \leq C \epsilon^{p-2}\left\|v_{\lambda, \epsilon, \sigma, y}\right\|_{\epsilon}+C\left(\epsilon+\sum_{k \neq j} \mathrm{e}^{-\frac{p-1}{p}\left|y_{k}-y_{j}\right|}\right) \\
& \leq \epsilon^{1-\tau}+\sum_{k \neq j} \mathrm{e}^{-\frac{p-1-\tau}{p}\left|y_{k}-y_{j}\right|} .
\end{aligned}
$$

Thus, $A_{y}$ maps $S_{y}$ into $S_{y}$ if $2<p \leq 3$. 
Suppose that $p>3$. Note that for any $a \in \mathbb{C},|\operatorname{Re} a| \leq|a|$. Then by Lemma A.4, Hölder inequality and the Sobolev inequality, we get

$$
\begin{aligned}
\left|\left\langle R_{y}^{\prime}\left(v_{\lambda, \epsilon, \sigma, y}\right), \xi\right\rangle\right|= & \operatorname{Re} \int\left|z_{y}+v_{\lambda, \epsilon, \sigma, y}\right|^{p-2} \overline{\left(z_{y}+v_{\lambda, \epsilon, \sigma, y}\right)} \xi-\operatorname{Re} \int\left|z_{y}\right|^{p-2} \bar{z}_{y} \xi \\
& -\operatorname{Re} \int\left[(p-2)\left|z_{y}\right|^{p-4} \operatorname{Re}\left(\bar{z}_{y} v_{\lambda, \epsilon, \sigma, y}\right) \bar{z}_{y} \xi+\left|z_{y}\right|^{p-2} \bar{v}_{\lambda, \epsilon, \sigma, y} \xi\right] \mid \\
\leq & \int|| z_{y}+\left.v_{\lambda, \epsilon, \sigma, y}\right|^{p-2} \overline{\left(z_{y}+v_{\lambda, \epsilon, \sigma, y}\right)}-\left|z_{y}\right|^{p-2} \bar{z}_{y} \\
& -\left[(p-2)\left|z_{y}\right|^{p-4} \operatorname{Re}\left(\bar{z}_{y} v_{\lambda, \epsilon, \sigma, y}\right) \bar{z}_{y}+\left|z_{y}\right|^{p-2} \bar{v}_{\lambda, \epsilon, \sigma, y}\right]|| \xi \mid \\
\leq & C \int\left|z_{y}\right|^{p-3}\left|v_{\lambda, \epsilon, \sigma, y}\right|^{2}|\xi| \\
\leq & C\left[\int\left(\left|z_{y}\right|^{p-3}\left|v_{\lambda, \epsilon, \sigma, y}\right|^{2}\right)^{\frac{p}{p-1}}\right]^{\frac{p-1}{p}}\|\xi\|_{\epsilon} .
\end{aligned}
$$

Hence, we get

$$
\left\|R_{y}^{\prime}\left(v_{\lambda, \epsilon, \sigma, y}\right)\right\|_{\epsilon} \leq C\left[\int\left(\left|z_{y}\right|^{p-3}\left|v_{\lambda, \epsilon, \sigma, y}\right|^{2}\right)^{\frac{p}{p-1}}\right]^{\frac{p-1}{p}} .
$$

Since $z_{y}$ is bounded, we have

$$
\left\|R_{y}^{\prime}\left(v_{\lambda, \epsilon, \sigma, y}\right)\right\|_{\epsilon} \leq C\left(\int\left|v_{\lambda, \epsilon, \sigma, y}\right|^{\frac{2 p}{p-1}}\right)^{\frac{p-1}{p}} \leq C\left\|v_{\lambda, \epsilon, \sigma, y}\right\|_{\epsilon}^{2} .
$$

For the estimate of $\left\|R_{y}^{\prime \prime}\left(v_{\lambda, \epsilon, \sigma, y}\right)\right\|_{\epsilon}$, by Hölder inequality and the Sobolev inequality, we have

$$
\begin{aligned}
\left|R_{y}^{\prime \prime}\left(v_{\lambda, \epsilon, \sigma, y}\right)(\xi, \vartheta)\right|= & \operatorname{Re} \int\left[(p-2)\left|z_{y}\right|^{p-4} \operatorname{Re}\left(\overline{z_{y}+v_{\lambda, \epsilon, \sigma, y}} \xi\right) \bar{z}_{y} \vartheta+\left|z_{y}\right|^{p-2} \bar{\xi} \vartheta\right] \\
& -\operatorname{Re} \int\left[(p-2)\left|z_{y}\right|^{p-4} \operatorname{Re}\left(\bar{z}_{y} \xi\right) \bar{z}_{y} \vartheta+\left|z_{y}\right|^{p-2} \bar{\xi} \vartheta\right] \mid \\
= & \operatorname{Re} \int(p-2)\left|z_{y}\right|^{p-4} \operatorname{Re}\left(\bar{v}_{\lambda, \epsilon, \sigma, y} \xi\right) \bar{z}_{y} \vartheta \mid \\
\leq & \int(p-2)\left|z_{y}\right|^{p-3}\left|v_{\lambda, \epsilon, \sigma, y}\right||\xi||\vartheta| \\
\leq & C\left(\int\left|v_{\lambda, \epsilon, \sigma, y}\right|^{3}\right)^{\frac{1}{3}}\left(\int|\xi|^{3}\right)^{\frac{1}{3}}\left(\int|\vartheta|^{3}\right)^{\frac{1}{3}} \\
\leq & C\left\|v_{\lambda, \epsilon, \sigma, y}\right\|_{\epsilon}\|\xi\|_{\epsilon}\|\vartheta\|_{\epsilon},
\end{aligned}
$$

which implies

$$
\left\|R_{y}^{\prime \prime}\left(v_{\lambda, \epsilon, \sigma, y}\right)\right\|_{\epsilon} \leq C\left\|v_{\lambda, \epsilon, \sigma, y}\right\|_{\epsilon} .
$$


Consequently, we have

$$
\begin{aligned}
\left\|A_{y}\left(v_{\lambda, \epsilon, \sigma, y}^{1}\right)-A\left(v_{\lambda, \epsilon, \sigma, y}^{2}\right)\right\|_{\epsilon} & =\left\|L_{y}^{-1} R_{y}^{\prime}\left(v_{\lambda, \epsilon, \sigma, y}^{1}\right)-L_{y}^{-1} R_{y}^{\prime}\left(v_{\lambda, \epsilon, \sigma, y}^{2}\right)\right\|_{\epsilon} \\
& \leq C\left\|R_{y}^{\prime}\left(v_{\lambda, \epsilon, \sigma, y}^{1}\right)-R_{y}^{\prime}\left(v_{\lambda, \epsilon, \sigma, y}^{2}\right)\right\|_{\epsilon} \\
& \leq C\left\|R_{y}^{\prime \prime}\left(\theta v_{\lambda, \epsilon, \sigma, y}^{1}+(1-\theta) v_{\lambda, \epsilon, \sigma, y}^{2}\right)\right\|_{\epsilon}\left\|v_{\lambda, \epsilon, \sigma, y}^{1}-v_{\lambda, \epsilon, \sigma, y}^{2}\right\|_{\epsilon} \\
& \leq C\left(\left\|v_{\lambda, \epsilon, \sigma, y}^{1}\right\|_{\epsilon}+\left\|v_{\lambda, \epsilon, \sigma, y}^{2}\right\| \|_{\epsilon}\right)\left\|v_{\lambda, \epsilon, \sigma, y}^{1}-v_{\lambda, \epsilon, \sigma, y}^{2}\right\|_{\epsilon} \\
& \leq \frac{1}{2}\left\|v_{\lambda, \epsilon, \sigma, y}^{1}-v_{\lambda, \epsilon, \sigma, y}^{2}\right\|_{\epsilon},
\end{aligned}
$$

where $0<\theta<1$ and

$$
\begin{aligned}
\left\|A_{y}\left(v_{\lambda, \epsilon, \sigma, y}\right)\right\|_{\epsilon} & =\left\|A_{y}\left(v_{\lambda, \epsilon, \sigma, y}\right)-A_{y}(0)\right\|_{\epsilon}+\left\|A_{y}(0)\right\|_{\epsilon} \\
& \leq C \epsilon\left\|v_{\lambda, \epsilon, \sigma, y}\right\|_{\epsilon}+C\left\|l_{y, k}\right\|_{\epsilon} \\
& \leq C \epsilon\left\|v_{\lambda, \epsilon, \sigma, y}\right\|_{\epsilon}+C\left(\epsilon+\sum_{k \neq j} \mathrm{e}^{-\frac{p-1}{p}\left|y_{k}-y_{j}\right|}\right) \\
& \leq \epsilon^{1-\tau}+\sum_{k \neq j} \mathrm{e}^{-\frac{p-1-\tau}{p}\left|y_{k}-y_{j}\right|} .
\end{aligned}
$$

Hence, $A_{y}$ is also a contraction map from $S_{y}$ to $S_{y}$. Now applying the contraction mapping theorem, for any $(y, \sigma) \in \Omega_{\lambda} \times[0,2 \pi]$, we can find a unique $v_{\lambda, \epsilon, \sigma, y}$ such that (2.7) holds. By (2.8) and (2.9), we obtain

$$
\left\|v_{\lambda, \epsilon, \sigma, y}\right\|_{\epsilon} \leq \epsilon^{1-\tau}+\sum_{k \neq j} \mathrm{e}^{-\frac{p-1-\tau}{p}\left|y_{k}-y_{j}\right|}
$$

To prove the $C^{1}$-continuity of $v_{\lambda, \epsilon, \sigma, y}$ with respect to $(y, \sigma)$, we can use the implicit function theorem to find a unique $C^{1}$-map: $(y, \sigma) \rightarrow \tilde{v}_{\lambda, \epsilon, y, \sigma}$, which solves (2.7) (see [11]). By the uniqueness, we see $v_{\lambda, \epsilon, \sigma, y}=\tilde{v}_{\lambda, \epsilon, y, \sigma}$ and hence is a $C^{1}$ map with respect to $(y, \sigma)$.

Finally, we prove $v_{\lambda, \epsilon, \sigma, y}=\mathrm{e}^{\mathrm{i} \sigma} V_{\lambda, \epsilon, y}$ with $V_{\lambda, \epsilon, y} \in E$ independent of $\sigma$. Since $v_{\lambda, \epsilon, \sigma, y}$ solves (2.7), from Lagrange multiplier theorem, there exist constants $X_{j} \in \mathbb{R}$ and $Y_{j} \in \mathbb{R}(j=1,2, \ldots, m)$ such that

$$
I_{\epsilon}^{\prime}\left(z_{y}+v_{\lambda, \epsilon, \sigma, y}\right)=\sum_{j=1}^{m} i X_{j} \mathrm{e}^{\mathrm{i} \sigma} w_{y_{j}}+\sum_{j=1}^{m} \sum_{\alpha=1}^{N} Y_{j} \mathrm{e} \frac{\partial w_{y_{j}}}{\partial x_{\alpha}} .
$$

Let $v_{\lambda, \epsilon, \sigma, y}=\mathrm{e}^{\mathrm{i} \sigma} V_{\lambda, \epsilon, y}$ with $V_{\lambda, \epsilon, y} \in E$. Noting that for any $\varphi \in E$

$$
\left\langle I_{\epsilon}^{\prime}(u), \varphi\right\rangle=\operatorname{Re} \int\left(\frac{\nabla}{i}-A_{\epsilon}(x)\right) \overline{\left(\frac{\nabla}{i}-A_{\epsilon}(x)\right) \varphi}+\operatorname{Re} \int V_{\epsilon}(x) u \bar{\varphi}-\operatorname{Re} \int|u|^{p-2} u \bar{\varphi}
$$

we test (2.10) by $\mathrm{e}^{\mathrm{i} \sigma} v(x)$ with $v(x)$ in $E$ and derive that $\sum_{j=1}^{N} w_{y_{j}}+V_{\lambda, \epsilon, y}$ is a solution of an equation independently of $\sigma$. Thus, $V_{\lambda, \epsilon, y}$ is independent of $\sigma$ and we complete the proof.

Lemma 2.3. If $\lambda \rightarrow \infty$ and for any $y \in \Omega_{\lambda}$, then

$$
\left|l_{y, k}\left(v_{\lambda, \epsilon, \sigma, y}\right)\right| \leq C\left(\epsilon+\sum_{k \neq j} \mathrm{e}^{-\frac{p-1}{p}\left|y_{k}-y_{j}\right|}\right)\left\|v_{\lambda, \epsilon, \sigma, y}\right\|_{\epsilon} .
$$


Proof. By Lemmas A.6 and A.8, we have

$$
\begin{aligned}
& \left.\left|\operatorname{Re} \int\right| z_{y}\right|^{p-2} z_{y} \bar{v}_{\lambda, \epsilon, \sigma, y}-\left.\sum_{j=1}^{m} \operatorname{Re} \int\left|\eta w_{y_{j}}\right|^{p-2} \eta w_{y_{j}} \bar{v}_{\lambda, \epsilon, \sigma, y}\left|\leq \int\right| z_{y}\right|^{p-2} z_{y}-\sum_{j=1}^{m}\left|\eta w_{y_{j}}\right|^{p-2} \eta w_{y_{j}}|| \bar{v}_{\lambda, \epsilon, \sigma, y} \mid \\
& \left.\leq\left.\int|| z_{y}\right|^{p-2} z_{y}-\left.\sum_{j=1}^{m}\left|\eta w_{y_{j}}\right|^{p-2} \eta w_{y_{j}}\right|^{\frac{p}{p-1}}\right)^{\frac{p-1}{p}}\left(\int\left|\bar{v}_{\lambda, \epsilon, \sigma, y}\right|^{p}\right)^{\frac{1}{p}} \\
& \leq C\left(\sum_{k \neq j} \int w_{y_{k}}^{p-1} w_{y_{j}}\right)^{\frac{p-1}{p}}\left\|v_{\lambda, \epsilon, \sigma, y}\right\|_{\epsilon} \\
& \leq C \sum_{k \neq j} \mathrm{e}^{-\frac{p-1}{p}\left|y_{k}-y_{j}\right|}\left\|v_{\lambda, \epsilon, \sigma, y}\right\|_{\epsilon},
\end{aligned}
$$

as $\lambda \rightarrow \infty$.

On the other hand, we have

$$
\begin{aligned}
\left|\epsilon \operatorname{Re} \int \frac{\nabla}{i} z_{y} B(x) \bar{v}_{\lambda, \epsilon, \sigma, y}\right| & \leq C \epsilon \int\left(\left|\frac{\nabla}{i} z_{y}\right|^{2}|B(x)|^{2}\right)^{\frac{1}{2}}\left(\int\left|v_{\lambda, \epsilon, \sigma, y}\right|^{2}\right)^{\frac{1}{2}} \\
& \leq C \epsilon \int\left(\left|\nabla z_{y}\right|^{2}|B(x)|^{2}\right)^{\frac{1}{2}}\left\|v_{\lambda, \epsilon, \sigma, y}\right\|_{\epsilon} \\
& \leq C \epsilon\left\|v_{\lambda, \epsilon, \sigma, y}\right\|_{\epsilon} .
\end{aligned}
$$

Similarly, we can get

$$
\begin{aligned}
\left|\epsilon \operatorname{Re} \int B(x) z_{y} \frac{\bar{\nabla}}{i} v_{\lambda, \epsilon, \sigma, y}\right| & \leq C \epsilon \int\left(\left|z_{y}\right|^{2}|B(x)|^{2}\right)^{\frac{1}{2}}\left\|v_{\lambda, \epsilon, \sigma, y}\right\|_{\epsilon} \leq C \epsilon\left\|v_{\lambda, \epsilon, \sigma, y}\right\|_{\epsilon}, \\
\left.\left|\epsilon^{2} \operatorname{Re} \int\right| B(x)\right|^{2} z_{y} \bar{v}_{\lambda, \epsilon, \sigma, y} \mid & \leq C \epsilon^{2} \int\left(\left|z_{y}\right|^{2}|B(x)|^{2}\right)^{\frac{1}{2}}\left\|v_{\lambda, \epsilon, \sigma, y}\right\|_{\epsilon} \\
& \leq C \epsilon^{2}\left\|v_{\lambda, \epsilon, \sigma, y}\right\|_{\epsilon} \leq C \epsilon\left\|v_{\lambda, \epsilon, \sigma, y}\right\|_{\epsilon}
\end{aligned}
$$

and

$$
\left|\epsilon \operatorname{Re} \int a(x) z_{y} \bar{v}_{\lambda, \epsilon, \sigma, y}\right| \leq C \epsilon\left(\int a(x)\left|z_{y}\right|^{2}\right)^{\frac{1}{2}}\left\|v_{\lambda, \epsilon, \sigma, y}\right\|_{\epsilon} \leq C \epsilon\left\|v_{\lambda, \epsilon, \sigma, y}\right\|_{\epsilon} .
$$

From (2.11) to (2.15), we get

$$
\left\|l_{y, k}\right\|_{\epsilon} \leq C\left(\epsilon+\sum_{k \neq j} \mathrm{e}^{-\frac{p-1}{p}\left|y_{k}-y_{j}\right|}\right)\left\|v_{\lambda, \epsilon, \sigma, y}\right\|_{\epsilon},
$$

as $\lambda \rightarrow \infty$.

For any $y=\left(y_{1}, \ldots, y_{m}\right) \in \Omega_{\lambda}$, define

$$
f_{m, \epsilon}\left(y_{1}, y_{2}, \ldots, y_{m}\right)=I_{\epsilon}\left(z_{y}+v_{\lambda, \epsilon, \sigma, y}\right) .
$$

Since $v_{\lambda, \epsilon, \sigma, y}=\mathrm{e}^{\mathrm{i} \sigma} V_{\lambda, \epsilon, y}$ with $V_{\lambda, \epsilon, y} \in E$ independent of $\sigma$, we know that $I_{\epsilon}\left(z_{y}+v_{\lambda, \epsilon, \sigma, y}\right)$ does not depend on $\sigma$ either.

From Lemma 2.2, we derive the following result, whose proof is standard and thus is omitted (see for example, $[8,31])$. 
Lemma 2.4. For large enough $\lambda>0$ and small enough $\epsilon>0$, if $y^{0}=\left(y_{1}^{0}, \ldots, y_{m}^{0}\right) \in \Omega_{\lambda}$ is a critical point of $f_{m, \epsilon}$, then $z_{y^{0}}+v_{\lambda, \epsilon, \sigma, y^{0}}$ is a critical point of $I_{\epsilon}$.

\section{Proof of our Main Result}

In this section, we will prove Theorem 1.1. In order to prove it, first we prove that for $\epsilon>0$ small enough, we can choose $\mu=\mu(\epsilon)$ large enough such that the function $f_{m, \epsilon}\left(y_{1}, \ldots, y_{m}\right)$ defined in (2.16) attains its maximum in $\Omega_{\mu}$ at some point $y^{(0)}=\left(y_{1}^{(0)}, \ldots, y_{m}^{(0)}\right)$. We know that $z_{y^{(0)}}+v_{\lambda, \epsilon, \sigma, y^{(0)}}$ is a solution of (1.1) by Lemma 2.4. Here we mainly apply the technique in $[28,31]$, but we make some minor modifications.

Considering that the case $m=1$ is much easier, we will discuss the case $m \geq 2$. Define

$$
d=\sup _{y \in\left(\mathbb{R}^{N}\right)^{m}}\left\{\int a(x)\left|z_{y}\right|^{2}\right\}
$$

We choose a number $l$ such that $l>\max \left\{1,3 d C_{4}^{-1}\right\}$. Then for any $\epsilon$ satisfying

$$
0<\epsilon<\min \left\{\left(\frac{l C_{4}}{2 C_{4}^{\prime}}\right)^{\frac{p}{p-2-2 \tau}}, \frac{1}{l}|w|_{p}^{p}\right\},
$$

there exists $\mu^{*}=\mu^{*}(\epsilon)>\mu=\mu(\epsilon)>0$ such that, for $z \in \mathbb{R}^{N}$ with $|z| \in\left[\mu(\epsilon), \mu^{*}(\epsilon)\right]$,

$$
l \epsilon \leq \int w^{p-1}(x) w(x-z) \mathrm{d} x \leq 2 l \epsilon .
$$

Define

$$
M_{\epsilon}:=\sup \left\{f_{m, \epsilon}(y) \mid y \in \Omega_{\mu(\epsilon)}\right\} .
$$

Denote

$$
C_{0}=\frac{1}{2} \int\left[|\nabla(\eta w)|^{2}+|\eta w|^{2}\right]-\frac{1}{p} \int|\eta w|^{p} .
$$

In order to get an $m$-bump solution of (1.1), it is sufficient to prove that $M_{\epsilon}$ is achieved in the interior of $\Omega_{\mu(\epsilon)}$.

Lemma 3.1. Let $m \geq 2$. Then for $\epsilon>0$ sufficiently small,

$$
M_{\epsilon}>\sup \left\{f_{m, \epsilon}(y) \mid y \in \Omega_{\mu(\epsilon)} \text { and }\left|y_{k}-y_{j}\right| \in\left[\mu(\epsilon), \mu^{*}(\epsilon)\right] \text { for some } k \neq j\right\} .
$$

Proof. Observe that $\mu(\epsilon)=O\left(\ln \frac{1}{\epsilon}\right) \rightarrow \infty$ as $\epsilon \rightarrow 0$.

By Lemma 2.2 (ii) and (3.1) we know that if $y \in \Omega_{\mu(\epsilon)}$, then

$$
\left\|v_{\lambda, \epsilon, \sigma, y}\right\|_{\epsilon} \leq \epsilon^{1-\tau}+\sum_{k \neq j} \mathrm{e}^{-\frac{p-1-\tau}{p}\left|y_{k}-y_{j}\right|} \leq C_{1} \epsilon^{\frac{p-1-\tau}{p}} .
$$

Note that

$$
\begin{aligned}
\frac{1}{2}\left\langle L_{y} v_{\lambda, \epsilon, \sigma, y}, v_{\lambda, \epsilon, \sigma, y}\right\rangle= & \frac{1}{2} \int\left\{\left[\left|\left(\frac{\nabla}{i}-A_{\epsilon}(x)\right) v_{\lambda, \epsilon, \sigma, y}\right|^{2}+V_{\epsilon}(x)\left|v_{\lambda, \epsilon, \sigma, y}\right|^{2}\right]\right. \\
& \left.-\left[(p-2)\left|z_{y}\right|^{p-2}\left(\operatorname{Re}\left(z_{y} \bar{v}_{\lambda, \epsilon, \sigma, y}\right)\right)^{2}+\left|z_{y}\right|^{p-2}\left|v_{\lambda, \epsilon, \sigma, y}\right|^{2}\right]\right\} \\
\leq & \frac{1}{2}\left\|v_{\lambda, \epsilon, \sigma, y}\right\|_{\epsilon}^{2}+(p-1) \int\left|z_{y}\right|^{p-2}\left|v_{\lambda, \epsilon, \sigma, y}\right|^{2} \\
\leq & \frac{1}{2}\left\|v_{\lambda, \epsilon, \sigma, y}\right\|_{\epsilon}^{2}+C\left\|v_{\lambda, \epsilon, \sigma, y}\right\|_{\epsilon}^{2}=C_{2}\left\|v_{\lambda, \epsilon, \sigma, y}\right\|_{\epsilon}^{2}
\end{aligned}
$$


and by Lemma A.5

$$
\begin{aligned}
\left|R_{y}\left(v_{\lambda, \epsilon, \sigma, y}\right)\right|= & \mid \frac{1}{p} \int\left\{\left|z_{y}+v_{\lambda, \epsilon, \sigma, y}\right|^{p}-\left|z_{y}\right|^{p}-p\left[\left|z_{y}\right|^{p-2} \operatorname{Re}\left(z_{y} \bar{v}_{\lambda, \epsilon, \sigma, y}\right)\right]\right. \\
& \left.-\frac{p}{2}\left[(p-2)\left|z_{y}\right|^{p-4}\left(\operatorname{Re}\left(z_{y} \bar{v}_{\lambda, \epsilon, \sigma, y}\right)\right)^{2}+\left|z_{y}\right|^{p-2}\left|v_{\lambda, \epsilon, \sigma, y}\right|^{2}\right]\right\} \mid \\
\leq & C \int\left|v_{\lambda, \epsilon, \sigma, y}\right|^{p^{*}} \leq C\left\|v_{\lambda, \epsilon, \sigma, y}\right\|_{\epsilon}^{p^{*}} \leq C_{3}\left\|v_{\lambda, \epsilon, \sigma, y}\right\|_{\epsilon}^{2},
\end{aligned}
$$

where $p^{*}=\min \{3, p\}>2$.

Hence, by Lemma 2.3, we have

$$
\begin{aligned}
& f_{m, \epsilon}\left(y_{1}, y_{2}, \ldots, y_{m}\right)=I_{\epsilon}\left(z_{y}+v_{\lambda, \epsilon, \sigma, y}\right) \\
& =I_{\epsilon}\left(z_{y}\right)+l_{y}\left(v_{\lambda, \epsilon, \sigma, y}\right)+\frac{1}{2}\left\langle L_{y} v_{\lambda, \epsilon, \sigma, y}, v_{\lambda, \epsilon, \sigma, y}\right\rangle-R_{y}\left(v_{\lambda, \epsilon, \sigma, y}\right) \\
& =I_{\epsilon}\left(z_{y}\right)+O\left(\left\|l_{y}\right\|_{\epsilon}\left\|v_{\lambda, \epsilon, \sigma, y}\right\|_{\epsilon}+\left\|v_{\lambda, \epsilon, \sigma, y}\right\|_{\epsilon}^{2}\right) \\
& =I_{\epsilon}\left(z_{y}\right)+O\left(\epsilon^{\frac{2(p-1-\tau)}{p}}\right) \\
& =\frac{1}{2} \int\left|\left(\frac{\nabla}{i}-A_{\epsilon}(x)\right) z_{y}\right|^{2}+V_{\epsilon}(x)\left|z_{y}\right|^{2}-\frac{1}{p} \int\left|z_{y}\right|^{p}+O\left(\epsilon^{\frac{2(p-1-\tau)}{p}}\right) \\
& =\frac{1}{2} \int\left|\frac{\nabla}{i} z_{y}\right|^{2}+\left|z_{y}\right|^{2}+\frac{\epsilon}{2} \int a(x)\left|z_{y}\right|^{2}+\frac{\epsilon^{2}}{2} \int|B(x)|^{2}\left|z_{y}\right|^{2}-\epsilon \operatorname{Re} \int \frac{\nabla}{i} z_{y} B(x) \bar{z}_{y} \\
& -\frac{1}{p} \int\left|z_{y}\right|^{p}+O\left(\epsilon^{\frac{2(p-1-\tau)}{p}}\right) \\
& =\left\{\sum_{j=1}^{m} \frac{1}{2} \int\left|\frac{\nabla}{i}\left(\eta w_{y_{j}}\right)\right|^{2}+\left|\eta w_{y_{j}}\right|^{2}-\sum_{j=1}^{m} \int \frac{1}{p}\left|\eta w_{y_{j}}\right|^{p}\right\}+\frac{\epsilon}{2} \int a(x)\left|z_{y}\right|^{2} \\
& +\left\{\sum_{k<j} \operatorname{Re} \int \frac{\nabla}{i}\left(\eta w_{y_{k}}\right) \frac{\bar{\nabla}}{i}\left(\eta w_{y_{j}}\right)+\operatorname{Re} \sum_{k<j} \int \eta w_{y_{k}} \overline{\eta w_{y_{j}}}\right\} \\
& +\frac{1}{p} \sum_{j=1}^{m} \int\left|\eta w_{y_{j}}\right|^{p}-\frac{1}{p} \int\left|z_{y}\right|^{p}+O\left(\epsilon^{\frac{2(p-1-\tau)}{p}}\right) \\
& =m C_{0}+\left\{\sum_{k<j} \int w_{y_{j}}^{p-1} w_{y_{k}}-\frac{1}{p} \int u_{y}^{p}+\frac{1}{p} \sum_{j=1}^{m} \int w_{y_{j}}^{p}\right\}+\frac{\epsilon}{2} \int a(x)\left|z_{y}\right|^{2}+O\left(\epsilon^{\frac{2(p-1-\tau)}{p}}\right) \\
& =: m C_{0}+\frac{\epsilon}{2} \int a(x)\left|z_{y}\right|^{2}-\mathcal{L}_{y}
\end{aligned}
$$

where

$$
\mathcal{L}_{y}=-\sum_{k<j} \int w_{y_{j}}^{p-1} w_{y_{k}}+\frac{1}{p} \int u_{y}^{p}-\frac{1}{p} \sum_{j=1}^{m} \int w_{y_{j}}^{p}+O\left(\epsilon^{\frac{2(p-1-\tau)}{p}}\right) .
$$

Assume that $y=\left(y_{1}, \ldots, y_{m}\right) \in \Omega_{\mu(\epsilon)}$ and $\left|y_{k}-y_{j}\right| \in\left[\mu(\epsilon), \mu^{*}(\epsilon)\right]$ for some $k \neq j$.

On one hand, by Lemma A.7, we have

$$
\int u_{y}^{p}=\int\left(\sum_{j=1}^{m} w_{y_{j}}\right)^{p} \geq \sum_{j=1}^{m} \int w_{y_{j}}^{p}+2(p-1) \sum_{1 \leq k<j \leq m} \int w_{y_{k}}^{p-1} w_{y_{j}},
$$


where we use the fact that

$$
\int w_{y_{k}}^{p-1} w_{y_{j}}=\int w_{y_{j}}^{p-1} w_{y_{k}}
$$

Therefore, by (3.1),

$$
\mathcal{L}_{y} \geq C_{4} \sum_{1 \leq k<j \leq m} \int w_{y_{k}}^{p-1} w_{y_{j}}-C_{4}^{\prime} \epsilon^{\frac{2(p-1-\tau)}{p}} \geq C_{4} l \epsilon-C_{4}^{\prime} \epsilon^{\frac{2(p-1-\tau)}{p}} \geq \frac{1}{2} C_{4} l \epsilon>\frac{3}{2} d \epsilon .
$$

Hence,

$$
\begin{aligned}
f_{m, \epsilon}\left(y_{1}, y_{2}, \ldots, y_{m}\right) & \leq m C_{0}+\frac{\epsilon}{2} \int a(x)\left|z_{y}\right|^{2}-\mathcal{L}_{y} \\
& \leq m C_{0}+\frac{d}{2} \epsilon-\frac{3}{2} d \epsilon=m C_{0}-d \epsilon .
\end{aligned}
$$

On the other hand, by Lemma A.3, we get

$$
\int u_{y}^{p}=\int\left(\sum_{j=1}^{m} w_{y_{j}}\right)^{p} \leq \sum_{j=1}^{m} \int w_{y_{j}}^{p}+C \sum_{1 \leq k<j \leq m} \int w_{y_{k}}^{p-1} w_{y_{j}},
$$

where we also use the fact that

$$
\int w_{y_{k}}^{p-1} w_{y_{j}}=\int w_{y_{j}}^{p-1} w_{y_{k}}
$$

Then we have

$$
\mathcal{L}_{y} \leq C_{5}\left(\sum_{k<j} \int w_{y_{k}}^{p-1} w_{y_{j}}\right)+O\left(\epsilon^{\frac{2(p-1-\tau)}{p}}\right)
$$

Hence

$$
\begin{aligned}
f_{m, \epsilon}\left(y_{1}, y_{2}, \ldots, y_{m}\right) & =m C_{0}+\frac{\epsilon}{2} \int a(x)\left|z_{y}\right|^{2}-\mathcal{L}_{y} \\
& \geq m C_{0}+\frac{\epsilon}{2} \int a(x)\left|z_{y}\right|^{2}-C_{6} \epsilon^{\frac{2(p-1-\tau)}{p}}+o(1),
\end{aligned}
$$

where o(1) denotes some quantities which depend only on $y$ and converge to 0 as $\left|y_{k}-y_{j}\right| \rightarrow \infty$ for all $k \neq j$. Hence, for $\epsilon>0$ sufficiently small,

$$
\liminf _{\left|y_{k}-y_{j}\right| \rightarrow \infty, \forall k \neq j} f_{m, \epsilon}\left(y_{1}, y_{2}, \ldots, y_{m}\right) \geq m c_{0} .
$$

Combining (3.4) and (3.5), we complete the proof of this lemma.

Choose $y^{(h)}(\epsilon)=\left(y_{1}^{(h)}(\epsilon), \ldots, y_{m}^{(h)}(\epsilon)\right) \in \Omega_{\mu(\epsilon)}$ such that

$$
\lim _{h \rightarrow \infty} f_{m, \epsilon}\left(y_{1}^{(h)}(\epsilon), \ldots, y_{m}^{(h)}(\epsilon)\right)=M_{\epsilon} .
$$

By Lemma 3.1, we may assume that

$$
\inf _{h} \min _{k \neq j}\left|y_{k}^{(h)}(\epsilon)-y_{j}^{(h)}(\epsilon)\right| \geq \mu^{*} .
$$


Thus, for any $1 \leq k \leq m$, passing to a subsequence if necessary, we may assume either $\lim _{h \rightarrow \infty} y_{k}^{(h)}(\epsilon)=y_{k}^{(0)}(\epsilon) \epsilon$ $\mathbb{R}^{N}$ with

$$
\left|y_{k}^{(0)}(\epsilon)-y_{j}^{(0)}(\epsilon)\right| \geq \mu^{*} \text { for } k \neq j \text { or } \lim _{h \rightarrow \infty}\left|y_{k}^{(h)}(\epsilon)\right|=\infty
$$

Define

$$
\Pi(\epsilon)=\left\{1 \leq k \leq m:\left|y_{k}^{(h)}(\epsilon)\right| \rightarrow \infty, \text { as } h \rightarrow \infty\right\} .
$$

We will prove that $\Pi(\epsilon)=\emptyset$ for $\epsilon>0$ small enough and hence $f_{m, \epsilon}$ attain its maximum at $\left(y_{1}^{(0)}(\epsilon), \ldots, y_{m}^{(0)}(\epsilon)\right)$ in $\Omega_{\mu(\epsilon)}$.

Lemma 3.2. Let $m \geq 2$. Then there exists $\epsilon(m)>0$ such that for any $\epsilon \in(0, \epsilon(m))$,

$$
\Pi(\epsilon)=\emptyset .
$$

Proof. We make a contradiction argument and assume that $\Pi(\epsilon) \neq \emptyset$ along a sequence $\epsilon_{n} \rightarrow 0$. Without loss of generality, we may assume $\Pi\left(\epsilon_{n}\right)=\left\{1,2, \ldots, j_{m}\right\}$ for all $n \in \mathbb{N}$ and for some $1 \leq j_{m}<m$. When $j_{m}=m$, we can hand by the same argument. For notation of simplicity, we will denote $\epsilon=\epsilon_{n}$ and $\left(y_{1}^{(h)}, y_{2}^{(h)}, \ldots, y_{m}^{(h)}\right)=$ $\left(y_{1}^{(h)}\left(\epsilon_{n}\right), y_{2}^{(h)}\left(\epsilon_{n}\right), \ldots, y_{m}^{(h)}\left(\epsilon_{n}\right)\right)$ for $h=0,1,2, \ldots$ Then, when $h \rightarrow \infty$

$$
\left|y_{1}^{(h)}\right| \rightarrow \infty, \ldots,\left|y_{j_{m}}^{(h)}\right| \rightarrow \infty \text { and } y_{j_{m}+1}^{(h)} \rightarrow y_{j_{m}+1}^{(0)}, \ldots, y_{m}^{(h)} \rightarrow y_{m}^{(0)} .
$$

Set

$$
y^{(h)}=\left(y_{1}^{(h)}, y_{2}^{(h)}, \ldots, y_{m}^{(h)}\right) \text { and } y_{*}^{(h)}=\left(y_{j_{m}+1}^{(h)}, y_{j_{m}+2}^{(h)}, \ldots, y_{m}^{(h)}\right)
$$

Let

$$
z_{h}=\sum_{k=1}^{m} \eta w_{y_{k}^{(h)}}:=\eta u_{h}, z_{h, 1}=\sum_{k=1}^{j_{m}} \eta w_{y_{k}^{(h)}}:=\eta u_{h, 1}, z_{h, 2}=\sum_{k=j_{m}+1}^{m} \eta w_{y_{k}^{(h)}}:=\eta u_{h, 2} .
$$

Similar to (3.2), we get

$$
\left\|v_{\mu, \epsilon, \sigma, y_{*}^{(h)}}\right\|_{\epsilon} \leq C_{7} \epsilon^{\frac{p-1-\tau}{p}},\left\|v_{\mu, \epsilon, \sigma, y^{(h)}}\right\|_{\epsilon} \leq C_{7} \epsilon^{\frac{p-1-\tau}{p}} .
$$

Now we rewrite $f_{m, \epsilon}\left(y_{1}^{(h)}, \ldots, y_{m}^{(h)}\right)$ as

$$
\begin{aligned}
f_{m, \epsilon}\left(y_{1}^{(h)}, \ldots, y_{m}^{(h)}\right)= & I_{\epsilon}\left(z_{h}+v_{\mu, \epsilon, \sigma, y^{(h)}}\right) \\
= & m C_{0}+\frac{\epsilon}{2} \int a(x)\left|z_{h}\right|^{2}-\mathcal{L}_{y^{(h)}} \\
= & j_{m} C_{0}+\frac{\epsilon}{2} \int a(x)\left|z_{h, 1}\right|^{2}+\left[\left(m-j_{m}\right) C_{0}+\frac{\epsilon}{2} \int a(x)\left|z_{h, 2}\right|^{2}-\mathcal{L}_{y_{*}^{(h)}}\right] \\
& +\epsilon \operatorname{Re} \int a(x) z_{h, 1} \bar{z}_{h, 2}+\mathcal{L}_{y_{*}^{(h)}}-\mathcal{L}_{y^{(h)}} .
\end{aligned}
$$

Since $\left|y_{k}^{(h)}\right| \rightarrow \infty, k=1, \ldots, j_{m}$, we get, as $h \rightarrow \infty$,

$$
\left.\left|\frac{\epsilon}{2} \int a(x)\right| z_{h, 1}\right|^{2}+\epsilon \operatorname{Re} \int a(x) z_{h, 1} \bar{z}_{h, 2} \mid \rightarrow 0 .
$$

From (3.7) and (3.8), we obtain

$$
f_{m, \epsilon}\left(y^{(h)}\right) \leq j_{m} C_{0}+f_{m-j_{m}, \epsilon}\left(y_{*}^{(h)}\right)+\mathcal{L}_{y_{*}^{(h)}}-\mathcal{L}_{y^{(h)}}+o(1) .
$$


By (3.3), we infer that

$$
\mathcal{L}_{y^{(h)}}=-\sum_{k<j} \int w_{y_{j}^{(h)}}^{p-1} w_{y_{k}^{(h)}}+\frac{1}{p} \int u_{h}^{p}-\sum_{j=1}^{m} \frac{1}{p} \int w_{y_{j}^{(h)}}^{p}+O\left(\epsilon^{\frac{2(p-1-\tau)}{p}}\right)
$$

and

$$
\mathcal{L}_{y_{*}^{(h)}}=-\sum_{j_{m}<k<j} \int w_{y_{j}^{(h)}}^{p-1} w_{y_{k}^{(h)}}+\frac{1}{p} \int u_{h, 2}^{p}-\sum_{j=1}^{m} \frac{1}{p} \int w_{y_{j}^{(h)}}^{p}+O\left(\epsilon^{\frac{2(p-1-\tau)}{p}}\right) .
$$

Then, by (3.10) and (3.11),

$$
\begin{aligned}
\mathcal{L}_{y_{*}^{(h)}}-\mathcal{L}_{y^{(h)}} & =\sum_{k<j \leq j_{m}} \int w_{y_{k}^{(h)}}^{p-1} w_{y_{j}^{(h)}}+\sum_{k=1}^{j_{m}} \int w_{y_{k}^{(h)}}^{p-1} u_{h, 2}+\frac{1}{p} \sum_{k=1}^{j_{m}} \int w_{y_{k}^{(h)}}^{p}+\frac{1}{p} \int u_{h, 2}^{p}-\frac{1}{p} \int u_{h}^{p}+O\left(\epsilon^{\frac{2(p-1-\tau)}{p}}\right) \\
& <O\left(\epsilon^{\frac{2(p-1-\tau)}{p}}\right) .
\end{aligned}
$$

Letting $h \rightarrow \infty$ in (3.9), we get

$$
M_{\epsilon} \leq j_{m} C_{0}+f_{m-j_{m}, \epsilon}\left(y_{j_{m}+1}^{(0)}, \ldots, y_{m}^{(0)}\right)+C_{8} \epsilon^{\frac{2(p-1-\tau)}{p}} .
$$

Furthermore, by Lemma A.8 and (3.1), we see that

$$
C_{9} \epsilon \leq \mu^{-\frac{N-1}{2}} \mathrm{e}^{-\mu} \leq C_{10} \epsilon
$$

which means that

$$
\frac{2}{3} \ln \frac{1}{\epsilon}<\mu=\mu(\epsilon)<2 \ln \frac{1}{\epsilon},
$$

for $\epsilon>0$ sufficiently small. Choose $\delta$ such that $0<\delta<\frac{p-2-2 \tau}{14 m p}$. By $\left(H_{1}\right)$, there exists $T>0$ such that

$$
a(x) \geq \mathrm{e}^{-\delta|x|}, \quad|x| \geq T .
$$

For $\epsilon>0$ sufficiently small, define

$$
\tilde{y}_{s}^{\epsilon}=\left(14 m \ln \epsilon^{-1}-6 s \mu(\epsilon)-1,0, \ldots, 0\right) \in \mathbb{R}^{N}, s=1,2, \ldots, m .
$$

The open balls $B\left(\tilde{y}_{s}^{\epsilon}, 3 \mu(\epsilon)\right)$ are mutually disjoint. Therefore there are $j_{m}$ integers from $\{1,2, \ldots, m\}$, denoted by $s_{1}<s_{2}<\cdots<s_{j_{m}}$, such that

$$
\left|\tilde{y}_{s_{k}}^{\epsilon}-y_{j}^{(0)}\right| \geq 3 \mu(\epsilon), k=1,2, \ldots, j_{m}, j=j_{m}+1, \ldots, m .
$$

Denote $\tilde{y}_{s_{k}}^{\epsilon}$ by $y_{k}^{\epsilon}, k=1,2, \ldots, j_{m}$. It follows that from (3.16)-(3.18), for $\epsilon>0$ small enough,

$$
\begin{gathered}
T+1 \leq\left|y_{k}^{\epsilon}\right| \leq 14 m \ln \epsilon^{-1}-1, k=1,2, \ldots, j_{m}, \\
\left|y_{k}^{\epsilon}-y_{j}^{\epsilon}\right| \geq 3 \mu(\epsilon), 1 \leq k<j \leq j_{m}, \\
\left|y_{k}^{\epsilon}-y_{j}^{(0)}\right| \geq 3 \mu(\epsilon), k=1,2, \ldots, j_{m}, j=j_{m}+1, \ldots, m .
\end{gathered}
$$

Thus,

$$
\left(y_{1}^{\epsilon}, \ldots, y_{j_{m}}^{\epsilon}, y_{j_{m}+1}^{(0)}, \ldots, y_{m}^{(0)}\right) \in \Omega_{\mu(\epsilon)} .
$$


Denote $y^{(\epsilon)}=\left(y_{1}^{\epsilon}, \ldots, y_{j_{m}}^{\epsilon}, y_{j_{m}+1}^{(0)}, \ldots, y_{m}^{(0)}\right)$ and $y_{*}^{(\epsilon)}=\left(y_{j_{m}+1}^{0}, \ldots, y_{m}^{0}\right)$. Let

$$
z_{\epsilon, 1}=\sum_{k=1}^{j_{m}} \eta w_{y_{k}^{\epsilon}}:=\eta u_{1, \epsilon} \text { and } z_{\epsilon, 2}=\sum_{k=j_{m}+1}^{m} \eta w_{y_{k}^{(0)}}=\eta u_{2, \epsilon}
$$

Similar to (3.7), we get

$$
\begin{aligned}
f_{m, \epsilon}\left(y_{1}^{\epsilon}, \ldots, y_{j_{m}}^{\epsilon}, y_{j_{m}+1}^{(0)}, \ldots, y_{m}^{(0)}\right)= & j_{m} C_{0}+f_{m-j_{m}, \epsilon}\left(y_{j_{m}+1}^{(0)}, \ldots, y_{m}^{(0)}\right)+\frac{\epsilon}{2} \int a(x)\left|z_{\epsilon, 1}\right|^{2}+\epsilon \operatorname{Re} \int a(x) z_{\epsilon, 1} \bar{z}_{\epsilon, 2} \\
& +\mathcal{L}_{y_{*}^{(\epsilon)}}-\mathcal{L}_{y^{(\epsilon)}} .
\end{aligned}
$$

Similar to (3.12), we have

$$
\begin{aligned}
\mathcal{L}_{y_{*}^{(\epsilon)}}-\mathcal{L}_{y^{(\epsilon)}} & =\sum_{k<j \leq j_{m}} \int w_{y_{k}^{(\epsilon)}}^{p-1} w_{y_{j}^{(\epsilon)}}+\sum_{k=1}^{j_{m}} \int w_{y_{k}^{p(\epsilon)}}^{p-1} u_{\epsilon, 2}+\frac{1}{p} \sum_{k=1}^{j_{m}} \int w_{y_{k}^{(\epsilon)}}^{p}+\frac{1}{p} \int u_{\epsilon, 2}^{p}-\frac{1}{p} \int u_{\epsilon}^{p}+O\left(\epsilon^{\frac{2(p-1-\tau)}{p}}\right) \\
& \geq \frac{1}{p} \sum_{k=1}^{j_{m}} \int w_{y_{k}^{(\epsilon)}}^{p}+\frac{1}{p} \int u_{\epsilon, 2}^{p}-\frac{1}{p} \int u_{\epsilon}^{p}+O\left(\epsilon^{\frac{2(p-1-\tau)}{p}}\right) .
\end{aligned}
$$

Then, by Lemma A.3, we get

$$
\mathcal{L}_{y_{*}^{(\epsilon)}}-\mathcal{L}_{y^{(\epsilon)}} \geq-C \sum_{k=1}^{j_{m}} \int w_{y_{k}^{p(\epsilon)}}^{p-1} u_{\epsilon, 2}-C \sum_{k=1}^{j_{m}} \int u_{\epsilon, 2}^{p-1} w_{y_{k}^{(\epsilon)}}-C \sum_{1 \leq k<j \leq j_{m}} \int w_{y_{k}^{p-1}}^{p-1} w_{y_{j}^{(\epsilon)}}+O\left(\epsilon^{\frac{2(p-1-\tau)}{p}}\right) .
$$

By Lemma A.8, (3.14) and (3.20), we have

$$
\sum_{1 \leq k<j \leq j_{m}} \int w_{y_{k}^{(\epsilon)}}^{p-1} w_{y_{j}^{(\epsilon)}}=o\left(\mathrm{e}^{-3 \mu}\right)=o\left(\epsilon^{2}\right), \text { as } \epsilon \rightarrow 0 .
$$

According to (3.21), a similar argument shows that

$$
\sum_{k=1}^{j_{m}} \int w_{y_{k}^{(\epsilon)}}^{p-1} u_{\epsilon, 2}+\sum_{k=1}^{j_{m}} \int u_{\epsilon, 2}^{p-2} w_{y_{k}^{(\epsilon)}}=o\left(\epsilon^{2}\right)
$$

From (3.24) to (3.25), we get

$$
\mathcal{L}_{y_{*}^{(\epsilon)}}-\mathcal{L}_{y^{(\epsilon)}} \geq O\left(\epsilon^{\frac{2(p-1-\tau)}{p}}\right)
$$

which with (3.22) yields

$$
\begin{aligned}
f_{m, \epsilon}\left(y_{1}^{\epsilon}, \ldots, y_{j_{m}}^{\epsilon}, y_{j_{m}+1}^{(0)}, \ldots,{ }_{m}^{(0)}\right) \geq & j_{m} C_{0}+f_{m-j_{m}, \epsilon}\left(y_{j_{m}+1}^{(0)}, \ldots, y_{m}^{(0)}\right)+\frac{\epsilon}{2} \int a(x)\left|z_{\epsilon, 1}\right|^{2} \\
& +\epsilon \operatorname{Re} \int a(x) z_{\epsilon, 1} \bar{z}_{\epsilon, 2}-C_{11} \epsilon^{\frac{2(p-1-\tau)}{p}} .
\end{aligned}
$$

By (3.16) and (3.19), we have, for $k=1,2, \ldots, j_{m}$,

$$
\begin{aligned}
\int a(x)\left|z_{\epsilon, 1}\right|^{2} & =\int a(x) u_{\epsilon, 1}^{2} \geq \int_{\left|x-y_{k}^{\epsilon}\right| \leq 1} a(x) u_{\epsilon, 1}^{2} \geq \int_{\left|x-y_{k}^{\epsilon}\right| \leq 1} \mathrm{e}^{-\delta|x|} u_{\epsilon, 1}^{2} \\
& \geq C_{12} \mathrm{e}^{-\delta\left(\left|y_{k}^{\epsilon}\right|+1\right)} \geq C_{12} \mathrm{e}^{-14 m \delta \ln \epsilon^{-1}}=C_{12} \epsilon^{14 m \delta} .
\end{aligned}
$$


Hence,we get for $\epsilon$ small enough,

$$
\begin{aligned}
f_{m, \epsilon}\left(y_{1}^{\epsilon}, \ldots, y_{j_{m}}^{\epsilon}, y_{j_{m}+1}^{(0)}, \ldots,{ }_{m}^{(0)}\right) & \geq j_{m} C_{0}+f_{m-j_{m}, \epsilon}\left(y_{j_{m}+1}^{(0)}, \ldots, y_{m}^{(0)}\right)+C_{12} \epsilon^{14 m \delta+1}-C_{11} \epsilon^{\frac{2(p-1-\tau)}{p}} \\
& \geq j_{m} C_{0}+f_{m-j_{m}, \epsilon}\left(y_{j_{m}+1}^{(0)}, \ldots, y_{m}^{(0)}\right)+C_{13} \epsilon^{14 m \delta+1},
\end{aligned}
$$

since $14 m \delta+1<\frac{2(p-1-\tau)}{p}$. It contradicts to (3.13). Thus there exists $\epsilon(m)>0$ such that if $0<\epsilon<\epsilon(m)$, then $\Pi(\epsilon)=\emptyset$ and $f_{m, \epsilon}$ achieves its maximum at some point $\left(y_{1}^{(0)}, \ldots, y_{m}^{(0)}\right) \in \Omega_{\mu(\epsilon)}$.

We are now in position to prove Theorem 1.1.

Proof of Theorem 1.1. By Lemma 3.2, if $m \geq 2$ and $0<\epsilon<\epsilon(m)$, then $f_{m, \epsilon}$ achieves its maximum at some point $\left(y_{1}^{(0)}, \ldots, y_{m}^{(0)}\right) \in \Omega_{\mu(\epsilon)}$. Then $z_{y^{(0)}}+v_{\lambda, \epsilon, \sigma, y^{(0)}}$ is an $m$-bump complex-valued solution of (1.1). By Lemma 2.2 (ii), if $m=1$ and $\epsilon \in\left(0, \epsilon_{0}\right]$, then

$$
\lim _{|y| \rightarrow \infty} f_{m, \epsilon}(y)=\lim _{|y| \rightarrow \infty} I_{\epsilon}\left(z_{y}+v_{\lambda, \epsilon, \sigma, y}\right)=I_{0}(\eta w)=C_{0} .
$$

For $m=1$, since $f_{m, \epsilon}$ is defined on all $\mathbb{R}^{N}, f_{m, \epsilon}$ has a critical point $y^{(0)} \in \mathbb{R}^{N}$ and $z_{y^{(0)}}+v_{\lambda, \epsilon, \sigma, y^{(0)}}$ is a complex-valued solution of (1.1).

Set $\epsilon(1)=\epsilon_{0}$ and $\epsilon_{1}(m)=\min \{\epsilon(1), \epsilon(2) \ldots, \epsilon(m)\}$. If $0<\epsilon<\epsilon_{1}(m)$, then (1.1) has at least $m$ nontrivial complex-valued solutions.

\section{Appendix A}

Since the following two lemmas are very basic, we omit their proofs and one can refer [13].

Lemma A.1. For any $u \in E$, there exists $C>0$ such that

$$
\int\left|\left(\frac{\nabla}{i}-A_{\epsilon}(x)\right) u\right|^{2}+V_{\epsilon}(x)|u|^{2} \geq C \int\left(|\nabla u|^{2}+|u|^{2}\right) .
$$

Lemma A.2. The two norms $\|\cdot\|$ in $H^{1}\left(\mathbb{R}^{N}, \mathbb{C}\right)$ and $\|\cdot\|_{\epsilon}$ in $E$ are equivalent.

Now we give some elementary inequalities which are applied in the previous subsections. For these inequalities, one can refer [29-31].

Lemma A.3. For $q>1$, there exists $C>0$ such that for any $a, b \in \mathbb{C}$,

$$
|| a+\left.b\right|^{q}-|a|^{q}-\left.|b|^{q}|\leq C| a\right|^{q-1}|b|+C|a||b|^{q-1} .
$$

Lemma A.4. For $q>1$, there exists $C>0$ such that for any $a, b \in \mathbb{C}$ and $|a|>|b|$,

$$
|| a+\left.b\right|^{q}(\bar{a}+\bar{b})-|a|^{q} \bar{a}-\left.\left[q|a|^{q-2} \operatorname{Re}(\bar{a} b) \bar{a}+|a|^{q} \bar{b}\right]|\leq C| a\right|^{q-1}|b|^{2} .
$$

Lemma A.5. For $q>2$, there exists $C>0$ such that for any $a, b \in \mathbb{C}$ and $|a|>|b|$,

$$
\begin{aligned}
& || a+\left.b\right|^{q}-|a|^{q}-q|a|^{q-2} \operatorname{Re}(a \bar{b})-\frac{q}{2}\left[(q-2)|a|^{q-4}(\operatorname{Re}(a \bar{b}))^{2}+|a|^{q-2}|b|^{2}\right] \mid \\
& \leq\left\{\begin{array}{l}
C|a|^{q-3}|b|^{3}, \text { if } q>3, \\
C|a|^{3-q}|b|^{q}, \text { if } 2<q \leq 3 .
\end{array}\right.
\end{aligned}
$$


Lemma A.6. For $q \geq 2$ and $m \in \mathbb{N}$, there exists $C>0$ such that for any $a_{j} \in \mathbb{C}, j=1,2, \ldots, m$,

$$
\left.|| \sum_{j=1}^{m} a_{j}\right|^{q-2}\left(\sum_{j=1}^{m} a_{j}\right)-\left.\sum_{j=1}^{m}\left|a_{j}\right|^{q-2} a_{j}\right|^{\frac{q}{q-1}} \leq C \sum_{k \neq j}\left|a_{k}\right|^{q-1}\left|a_{j}\right| .
$$

Lemma A.7. For $q \geq 2$ and $m \in \mathbb{N}$, there exists $C>0$ such that for any $a_{j} \geq 0, j=1,2, \ldots, m$,

$$
\left(\sum_{j=1}^{m} a_{j}\right)^{q} \geq \sum_{j=1}^{m} a_{j}^{q}+(q-1) \sum_{1 \leq k \neq j \leq m} a_{k}^{q-1} a_{j} .
$$

Lemma A.8 (Lem. II.2, [4]). There exists a positive constants $c>0$ such that as $\left|y_{k}-y_{j}\right| \rightarrow \infty$,

$$
\int w_{y_{k}}^{p-1} w_{y_{j}} \sim c\left|y_{k}-y_{j}\right|^{-\frac{N-1}{2}} \mathrm{e}^{-\left|y_{k}-y_{j}\right|} .
$$

Acknowledgements. The authors thank sincerely Professors Shuangjie Peng and Gongbao Li for helpful discussions and suggestions. This paper was partially supported by NSFC (Nos. 11071092, 11071095) and the Ph.D. specialized grant of the Ministry of Education of China (20110144110001). C.H. Wang was also supported by the State Scholarship Fund of CSC (2011677017).

\section{REFERENCES}

[1] A. Ambrosetti and A. Malchiodi, Perturbation Methods and Semilinear Elliptic Problems on $\mathbb{R}^{n}$, Progress in Mathematics 240. Binkäuser, Verlag (2006).

[2] A. Ambrosetti, A. Malchiodi and S. Secchi, Multiplicity results for some nonlinear Schrödinger equations with potentials. Arch. Rational Mech. Anal. 159 (2001) 253-271.

[3] G. Arioli and A. Szulkin, A semilinear Schrödinger equation in the presence of a magnetic field. Arch. Rational Mech. Anal. 170 (2003) 277-295.

[4] A. Bahri and P.L. Lions, On the existence of a positive solution of semilinear elliptic equations in unbounded domains. Ann. Inst. H. Poincaré Anal. Non Linéaire 14 (1997) 365-413.

[5] T. Bartsch, E.N. Dancer and S. Peng, On multi-bump semi-classical bound states of nonlinear Schrödinger euqations with electromagnetic fields. Adv. Differential Equations 7 (2006) 781-812.

[6] R. Brummelhuisa, Expotential decay in the semi-classical limit for eigenfunctions of Schrödinger operators with magnetic fields and potentials which degenerate at infinity. Comm. Partial Differential Equations 16 (1991) 1489-1502.

[7] J. Byeon and Y. Oshita, Existence of multi-bump standing waves with a critcal frequency for nonlinear Schrödinger euqations. Comm. Partial Differential Equations 29 (2004) 1877-1904.

[8] D. Cao and H.P. Heinz, Uniquness of positive multi-bump bound states of nonlinear elliptic Schrödinger equations. Math. Z. 243 (2003) 599-642.

[9] D. Cao and E.S. Noussair, Multi-bump standing waves with a critical frequency for nonlinear Schrödinger equations. J. Differential Equations 203 (2004) 292-312.

[10] D. Cao and S. Peng, Multi-bump bound states of Schrödinger equations with a critical frequency. Math. Ann. 336 (2006) 925-948.

[11] D. Cao and Z. Tang, Existence and Uniqueness of multi-bump bound states of nonlinear Schrödinger equations with electromagnetic fields. J. Differential Equations 222 (2006) 381-424.

[12] S. Cingolani and M. Clapp, Intertwining semiclassical bound states to a nonlinear magnetic Schrödinger equation. Nonlinearity 22 (2009) 2309-2331.

[13] S. Cingolani and S. Secchi, Semiclassical limit for nonlinear Schrödinger equations with electromagnetic fields. J. Math. Anal. Appl. 275 (2002) 108-130.

[14] S. Cingolani and S. Secchi, Semiclassical states for NLS equations with magnetic potentials having polynomial growths. J. Math. Phys. 46 (2005) 053503.

[15] S. Cingolani, L. Jeanjean and S. Secchi, Multi-peak solutions for magnetic NLS equations without non-degeneracy conditions. ESAIM: COCV 15 (2009) 653-675.

[16] M. del Pino and P.L. Felmer, Local mountain passes for a semilinear elliptic problems in unbounded domains, Calc. Var. Partial Differential Equations 4 (1996) 121-137.

[17] M. del Pino and P.L. Felmer, Semi-classical states for nonlinear Schrödinger equations. J. Funct. Anal. 149 (1997) $245-265$. 
[18] M. del Pino and P.L. Felmer, Multi-peak bound states for nonlinear Schrödinger equations. Ann. Inst. H. Poincaré Anal. Non Linéaire 15 (1998) 127-149.

[19] M. del Pino and P.L. Felmer, Semi-classical states of nonlinear Schrödinger equations: a varational reduction method. Math. Ann. 324 (2002) 1-32.

[20] M. Esteban and P.L. Lions, Stationary solutions of nonlinear Schrödinger equations with an external magnetic field, Partial differential equations and the calculus of variations I, Progr. Nonlinear Differential Equations Appl. 1. Birkhäuser, Boston, MA (1989) 401-449.

[21] A. Floer and A. Weinstein, Nonspreading wave packets for the cubic Schrödinger equation with a bounded potential. J. Funct. Anal. 69 (1986) 397-408.

[22] B. Helffer, On spectral theory for Schrödinger operator with magnetic potentials. Spectral and scattering theory and applications, Adv. Stud. Pure Math. 23. Math. Soc. Japan, Tokyo (1994) 113-141.

[23] B. Helffer, Semiclassical analysis for Schrödinger operator with magnetic wells, Quasiclassical methods (Minneapolis, MN, 1995), IMA Vol. Math. Appl. 95. Springer, New York (1997) 99-114.

[24] B. Helffer and J. Sjöstrand, The tunnel effect for the Schrödinger equation with magnetic field. Ann. Scuola Norm. Sup. Pisa Cl. Sci. 14 (1987) 625-657.

[25] K. Kurata, Existence and semi-classical limit of the least energy solution to a nonlinear Schrödinger equation with electromagenetic fields. Nonlinear Anal. 41 (2000) 763-778.

[26] M.K. Kwong, Uniqueness of positive solutions of $\Delta u-u+u^{p}=0$ in $\mathbb{R}^{n}$. Arch. Rational Mech. Anal. 105 (1989) $243-266$.

[27] Y.Y. Li, On a singularly perturbed equation with Neumann boundary condition. Comm. Partial Differential Equations 23 (1998) 487-545.

[28] G. Li, S. Peng and C. Wang, Multi-bump solutions for the nonlinear Schrödinger-Poisson system. J. Math. Phys. 52 (2011) 053505.

[29] G. Li, S. Peng and C. Wang, Infinitely many solutions for nonlinear Schrödinger equations with electromagnetic fields. J. Differential Equations 251 (2011) 3500-3521.

[30] L. Lin and Z. Liu, Multi-bump solutions and multi-tower solutions for equations on $\mathbb{R}^{N}$. J. Funct. Anal. 257 (2009) $485-505$.

[31] L. Lin, Z. Liu and S. Chen, Multi-bump solutions for a semilinear Schrödinger equation. Indiana Univ. Math. J. 58 (2009) 1659-1689.

[32] Y.G. Oh, Existence of semiclassical bound states of nonlinear Schrödinger equations with potentials of the class $(V)_{a}$. Comm. Partial Differential Equations 14 (1989) 833-834.

[33] Y.G. Oh, On positive multi-bump bound states of nonlinear Schrödinger equations under multiple well potential. Commun. Math. Phys. 131 (1990) 223-253.

[34] P.H. Rabinowitz, On a class of nonlinear Schrödinger equations. Z. Angew. Math. Phys. 43 (1992) 270-291.

[35] C. Sulem and P.L. Sulem, The Nonlinear Schrödinger Equation, Self-Focusing and Wave Collapse, Applied Mathematical Sciences 139. Springer-Verlag, New York, Berlin, Heidelberg (1999).

[36] Z. Tang, Multi-bump bound states of nonlinear Schrödinger equations with electromagnetic fields and critical frequency. J. Differential Equations 245 (2008) 2723-2748.

[37] Z. Tang, Multiplicity of standing wave solutions of nonlinear Schrödinger equations with electromagnetic fields. Z. Angew. Math. Phys. 59 (2008) 810-833.

[38] X. Wang, On a concentration of positive bound states of nonlinear Schrödinger equations. Commun. Math. Phys. 153 (1993) 229-244.

[39] Z.Q. Wang, Existence and symmetry of multi-bump solutions for nonlinear Schrödinger equations. J. Differential Equations 159 (1999) 102-137.

[40] X. Wang and B. Zeng, On concentration of positive bound states of nonlinear Schrödinger equations with competing potential functions. SIAM J. Math. Anal. 28 (1997) 633-655. 